\title{
Numerical study on butterfly wings around inclusion based on damage evolution and semi-analytical method
}

\author{
Ye ZHOU ${ }^{1,2,}$, Caichao ZHU ${ }^{1}$, Xiaojin $\mathrm{CHEN}^{3}$, Wei YE ${ }^{3}$ \\ ${ }^{1}$ State Key Laboratory of Mechanical Transmissions, Chongqing University, Chongqing 400030, China \\ ${ }^{2}$ College of Aerospace Engineering, Chongqing University, Chongqing 400044, China \\ ${ }^{3}$ Chongqing Wangjiang Industrial Co., Chongqing 400071, China \\ Received: 08 May 2021 / Revised: 24 June 2021 / Accepted: 04 July 2021 \\ (C) The author(s) 2021.
}

\begin{abstract}
Butterfly wings are closely related to the premature failure of rolling element bearings. In this study, butterfly formation is investigated using the developed semi-analytical three-dimensional (3D) contact model incorporating inclusion and material property degradation. The 3D elastic field introduced by inhomogeneous inclusion is solved by using numerical approaches, which include the equivalent inclusion method (EIM) and the conjugate gradient method (CGM). The accumulation of fatigue damage surrounding inclusions is described using continuum damage mechanics. The coupling between the development of the damaged zone and the stress field is considered. The effects of the inclusion properties on the contact status and butterfly formation are discussed in detail. The model provides a potential method for quantifying material defects and fatigue behavior in terms of the deterioration of material properties.
\end{abstract}

Keywords: contact fatigue; butterfly wings; semi-analytical method; damage evolution

\section{Introduction}

The occurrence of premature failures due to white etching cracks (WECs) in rolling element bearings is a primary limitation to improving the service performance and reliability of advanced equipment, such as wind turbines, high-speed trains, and tunnel boring machines. It has been reported that failures can occur in as little as 5\%-20\% of the desired L10 life of bearings in wind turbine gearboxes (WTG) [1, 2]. WECs are closely related to butterflies, which are symbiotic microstructures that contain white etching areas (WEAs), cracks, and inclusions. As one of the most important initiators of subsurface cracks, butterflies are characterized by a central stress concentrator (material defects such as inclusions) and surrounding microstructural alterations [3], as shown in Fig. 1. WEAs appear in pairs as wings on either side of the inclusion at angles of $\pm 30^{\circ}-50^{\circ}$ to the rolling

* Corresponding author: Ye ZHOU, E-mail: zhouye@cqu.edu.cn direction [4, 5]. The formation and morphology of butterflies are strongly linked to the local stress state. The shape, size, rigidity, and distribution of inclusions may change the stress/strain behavior, thus affecting the initiation and evolution of butterflies [6,7].

Following the pioneering observations of butterflies

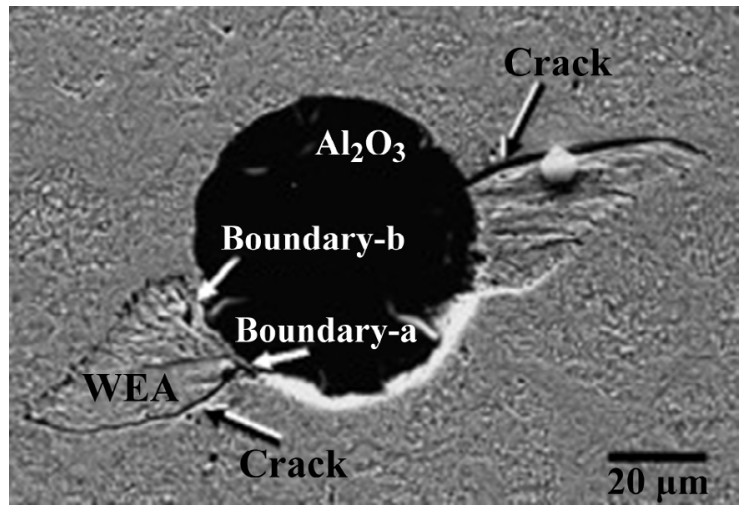

Fig. 1 Butterfly wings around inclusion. Reproduced with permission from Ref. [3], (C) Elsevier 2007. 
by Jones [8] and Styri [9], failure modes related to WECs and butterflies have been continuously investigated over the past decades. Evans et al. [10, 11] successfully reproduced the failure caused by WECs on a roller tribometer and a FAG-FE8 test rig. The evidence they obtained through the mapping and reconstruction of entire independent WECs using a combination of focused ion beam (FIB) and serial sectioning tomography techniques strongly supports the hypothesis that butterflies can be introduced by inclusions. In field bearing tests, bearings removed from a WTG were examined using X-ray microtomography, atomic force microscopy (AFM), and ultrasonic mapping. The crack morphology, microstructure alterations, and inclusions provide further support for the correlation between inclusions and butterflies [5, 6, 12-14]. The cracks are preferentially initiated at the central inclusion and propagated in the direction of over-rolling. The critical depth of the crack initiation has a strong correlation with the positions of the maximum shear stress. The factors influencing butterflies, such as kinematic sliding, lubricant composition, and material properties have been extensively investigated in benchtop tests using ball-on-rod or triple-disc rolling contact fatigue rigs [15-18]. The direction and magnitude of the sliding are highly related to the formation of butterflies. It has been reported that WECs and butterflies can be formed with synthetic commercial oils as well as base oils under both positive and negative sliding in various types of bearing steels $[19,20]$. Accordingly, butterflies, as a common type of rolling contact fatigue, should be further investigated numerically owing to their complicated stress/strain behavior.

A contact model that incorporates inclusion and damage evolution is necessary to understand the fundamental damage mechanisms of butterflies. This is the primary requirement for a numerical simulation of butterflies that solves for the subsurface stress field surrounding the inclusion and describes the microstructural alteration accompanying damage evolution. In early attempts made by Salehizadeh and Saka [21], Melander [22], and Yamakawa et al. [23], the local stress/strain fields around the inclusion were calculated using the finite element method (FEM). Several different types of inclusions (manganese sulfide, alumina, and titanium nitride) under rolling contact conditions were investigated. Raje et al. [24] proposed a discrete element approach to compute the elastic stress in an inhomogeneous medium. Ueda et al. [25] analytically predicted the location and direction of the initial crack emanating from an inclusion. Following McDowell's pioneering work [26], Alley and Neu [27] developed a numerical model of crystal plasticity using FEM. This influential simulation framework laid the foundation necessary to determine the effect of the microstructure in rolling contact fatigue (RCF). Based on this framework, the microstructural properties, including anisotropy, inclusion, and phase state, have been extensively studied [28-30]. Cerullo and Tvergaard [31] obtained the stress field surrounding the inclusion and evaluated the contact fatigue using the DangVan multiaxial criterion. Moghaddam et al. [32, 33] investigated the formation of butterflies based on a FEM model and continuum damage mechanics (CDM). Guan et al. [34] studied the crack nucleation and propagation induced by metallic carbides using the Voronoi finite element model. To capture the stress variation around the inclusion, a sufficiently fine meshing scheme is necessary for the numerical model. This would result in a long computation time for the FEM calculation, especially for 3D contact under cyclic loading.

The recent development of semi-analytical methods (SAMs) provides an alternative way to simulate butterfly formation. In semi-analytical models, the computation domain is discretized into cuboidal elements with uniform stress and strain. The total stress is given by the superposition of the influence coefficient (IC) response of each element solved by an analytical method. Jacq et al. [35] and Nelias et al. [36, 37] developed a 3D elastoplastic contact model using SAM. The equivalent inclusion method (EIM) assumes that an inhomogeneous area can be simulated as an inclusion with an equivalent eigenstrain. Based on this idea, Zhou et al. [38] proposed a semi-analytical solution that describes the disturbance stress field caused by multiple inclusions. Liu et al. [39] provided explicit solutions of the elastic field caused by an arbitrarily-shaped inclusion based on Galerkin vectors. In recent years, this SAM framework incorporating EIM has been extensively applied to the contact problems of inhomogeneous materials, including lubrication [40], partial slip [41], distributed inhomogeneities [42], and viscoelastic contact [43]. The combination of SAM and discrete convolution and 
fast Fourier transformation (DC-FFT) algorithm laid the foundation for efficiently and accurately simulating the damage evolution around inclusions.

In this work, a coupled-damage model incorporating inhomogeneous inclusions and material property degradation is proposed to investigate butterfly formation. The elastic field introduced by the inclusion and deterioration of material properties are obtained using SAM and EIM. The fatigue damage accumulation caused by the concentrated stress is described by CDM. Furthermore, the effects of inclusion depth and orientation angle on butterfly formation are discussed in detail. The findings from this study provide further insights into the mechanisms underlying butterfly formation. Although this numerical model can be further improved, for example by including the thermal effect and crack propagation, it provides a new approach for quantifying the damage evolution around the inclusion.

\section{Methodology}

\subsection{Contact problem description}

The numerical model consists of four major components, as illustrated in Fig. 2. (i) The pressure and traction are determined using the compatibility conditions of the contact surfaces. (ii) The inhomogeneous inclusions (for both non-metallic inclusions and microstructural alteration) are replaced by an imaginary equivalent homogeneous inclusion with eigenstrain. (iii) The elastic field incorporating the disturbance stress and displacement due to the eigenstrain is obtained. (iv) The damage accumulation and elastic modulus variation around the inclusions are updated to represent the microstructural alteration. The influences of lubrication and roughness are neglected because only a limited amount of stress variation is introduced by the lubrication condition on or near the surface [44, 45]. The gradient of the hardness is not considered because the simulated material is through hardening bearing steel. The four components are described in the following sections.

The general contact problem is governed by the gap and load balance equations as Eqs. (1) and (2):

$$
h(x, y)=h_{0}+h_{\mathrm{m}}(x, y)+u_{3}(x, y)
$$

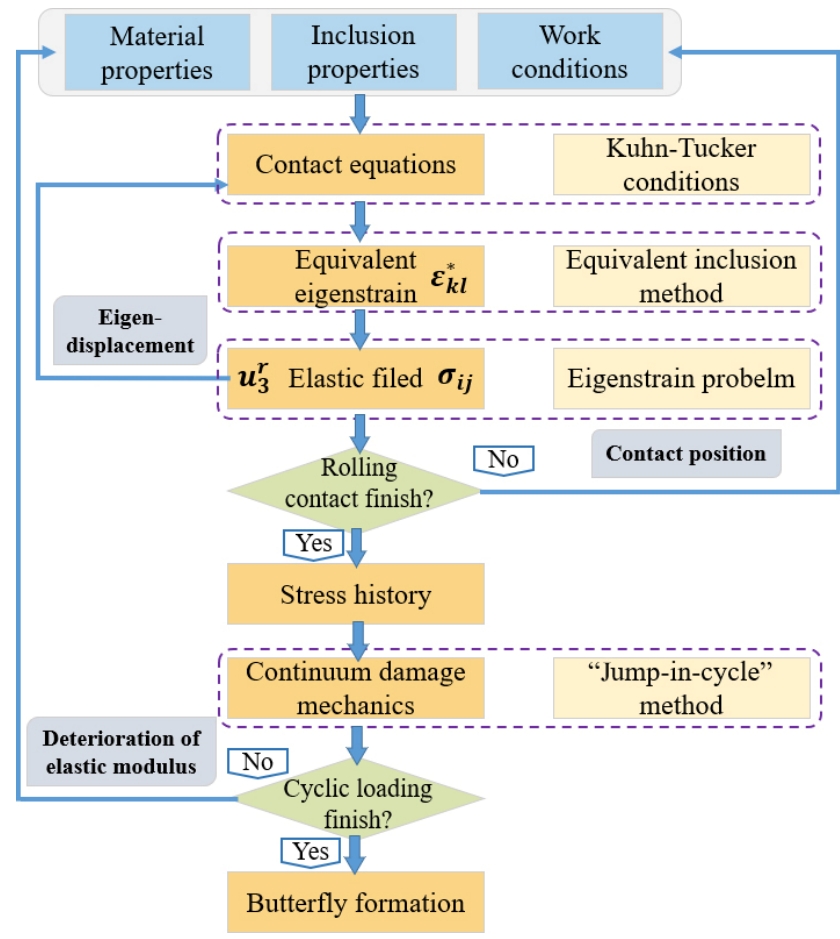

Fig. 2 Flow chart of the model.

$$
W_{\mathrm{n}}=\int_{\Gamma} p(x, y) \mathrm{d} \Gamma
$$

Here, $h(x, y)$ is the gap between the two contact surfaces; $h_{0}$ and $h_{\mathrm{m}}$ denote the rigid gap and initial macro-geometry, respectively; and $u_{3}$ is the surface normal displacement, which contains the elastic deformation $u_{3}^{\mathrm{e}}(x, y)$ and eigen-displacement $u_{3}^{\mathrm{r}}(x, y)$ in this work. Equation (2) represents the equilibrium of the surface pressure $p(x, y)$ and the normal load $W_{\mathrm{n}}$ in the contact area $\Gamma$. The boundary constraints are given as

$$
\begin{cases}p(x, y) \geq 0 & h(x, y)=0 \in \Gamma \\ p(x, y)=0 & h(x, y) \geq 0 \notin \Gamma\end{cases}
$$

The surface pressure can be solved based on the widely applied conjugate gradient method (CGM) [46]. The surface shear traction is simplified as the Coulomb friction. The coefficient of friction is obtained from the results of rings-to-roller tests [44].

\subsection{Equivalent inclusion method for inhomogeneous inclusion}

The inclusions and surrounding microstructural 
alterations can be treated as inhomogeneous inclusions because of their different elastic moduli [33]. As shown in Fig. 3, a deformable body with elastic modulus $C_{i j k l}$ contains several subdomains $\Omega_{\phi}(\phi=1,2,3 \ldots n)$, which are assumed to be perfectly bonded with the matrix and have different elastic moduli $C_{i j k l}^{\phi}$. The subdomains are, in general, subjected to the initial eigenstrain $\varepsilon_{k l}^{p}$ due to self-equilibration and the applied stress $\sigma_{i j}^{0}$ caused by an external load. The core idea in EIM is that an imaginary inclusion with the same elastic properties as the matrix can be enriched to simulate the influence of an inhomogeneous inclusion. This inclusion contains the initial eigenstrain $\varepsilon_{k l}^{p}$ and the equivalent eigenstrain $\varepsilon_{k l}^{*}$, which represents the disturbance caused by the inhomogeneity. Therefore, according to Hooke's law, the stress inside the subdomains can be expressed as

$$
\begin{gathered}
\sigma_{i j}=C_{i j k l}^{\phi}\left(\varepsilon_{k l}-\varepsilon_{k l}^{p}\right) \\
\sigma_{i j}=C_{i j k l}\left(\varepsilon_{k l}-\varepsilon_{k l}^{p}-\varepsilon_{k l}^{*}\right)
\end{gathered}
$$

Equations (4a) and (4b) indicate that the key step in EIM is to solve the equivalent eigenstrain $\varepsilon_{k l}^{*}$. Rewriting the total strain in Eq. (4a) as $\varepsilon_{i j}=C_{i j k l}^{\phi-1} \sigma_{k l}+\varepsilon_{k l}^{p}$, Eq. (4b) can also be written as

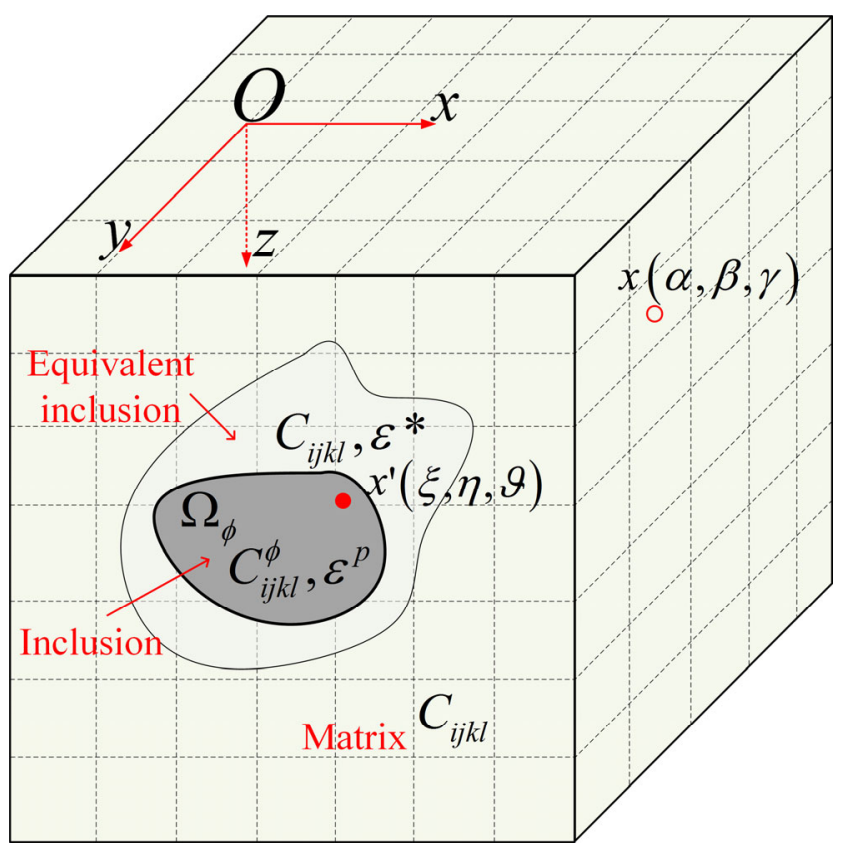

Fig. 3 Schematic of 3D inhomogeneous inclusion and equivalent inclusion.

$$
\sigma_{i j}=C_{i j k l}\left(C_{k l m n}^{\phi-1} \sigma_{m n}-\varepsilon_{m n}^{*}\right)
$$

The total stress can be determined as

$$
\sigma_{i j}=\sigma_{i j}^{*}+\sigma_{i j}^{0}+\sigma_{i j}^{p}
$$

where $\sigma_{i j}^{0}$ is the stress induced by the external load. $\sigma_{i j}^{p}$ and $\sigma_{i j}^{*}$ are the eigenstresses caused by the initial eigenstrain and equivalent eigenstrain, respectively. Equation (6) can be substituted into Eq. (5) to give the following:

$$
\sigma_{i j}^{*}-C_{i j k l}\left(C_{k l m n}^{\phi-1} \sigma_{m n}^{*}-\varepsilon_{m n}^{*}\right)=C_{i j k l} C_{k l m q}^{\phi-1}\left(\sigma_{m q}^{0}-\sigma_{m q}^{p}\right)-\sigma_{i j}^{0}-\sigma_{i j}^{p}
$$

Equation (7) can be written in matrix form as follows:

$$
\left(I-C C^{\phi-1}\right) \sigma^{*}+C \varepsilon^{*}=\left(C C^{\phi-1}-I\right)\left(\sigma^{p}+\sigma^{0}\right)
$$

In Eq. (8), the three unknowns are $\boldsymbol{\sigma}^{*}, \boldsymbol{\sigma}^{p}$, and $\boldsymbol{\varepsilon}^{*}$. The explicit expressions for the elastic constants, $C$ and $C^{\phi-1}$, are listed in Appendix A. In general, the mapping relationship from the eigenstrain to the eigenstress can be assumed to take the form of

$$
\sigma^{*}=\Upsilon\left(\varepsilon^{*}\right)
$$

Equation (8) can then be written as

$$
\left(I-C C^{\phi-1}\right) Y\left(\varepsilon^{*}\right)+C \varepsilon^{*}=\left(C C^{\phi-1}-I\right)\left[Y\left(\varepsilon^{p}\right)+\sigma^{0}\right]
$$

Once the operation $Y$ is determined, the governing equation of the equivalent eigenstrain becomes solvable, because $\varepsilon^{*}$ is the only unknown. In short, Eq. (10) can be simplified to a linear equation, that is, $\boldsymbol{F} \boldsymbol{\varepsilon}^{*}=\boldsymbol{a}$, for which the conjugate gradient method (CGM) can be used to obtain the solution. More details on the CGM and the solution of Eq. (10) can be found in Appendix B. A key part of this procedure, the operation $Y$ associated with the eigenstrain problem, is explained in the next section.

\subsection{Stress field and surface displacement}

As summarized by Mura [47], incompatibility of the eigenstrains results in the creation of eigenstress fields. The general solution of elastic fields related to eigenstrain has been investigated by Chiu [48], Jacq 
et al. [35], Nelias et al. [36], and Liu et al. [39] over the decades. A special form of this problem considering elastoplastic contact was also discussed by the author in Ref. [49]. In general, the eigenstrain can be treated as an excitation in the elastic field and the eigenstress as its response. Once the point-to-point response function has been derived, the total stress can be determined via the summation of every element. The elastic deformation due to eigenstrain can be expressed in terms of the Galerkin vectors, $\boldsymbol{F}$ [50]:

$$
2 \mu u_{i}^{r}(x)=2(1-v) F_{i, j j}-F_{k, k i}
$$

where $v$ is the Poisson's ratio, and $\mu$ is the Lamé constant. The commas in the subscripts indicate the derivatives with respect to the indices following the commas, e.g., $F_{i, j}=\partial F_{i} / \partial F_{j}$. Once the deformations are determined using Eq. (11), the eigenstress inside and outside the inclusion area $\Omega$ can be derived using Hooke's law:

$$
\begin{gathered}
\sigma_{i j}^{r}(x)=C_{i j k l}\left[\left(u_{k, l}+u_{l, k}\right) / 2\right] \quad \text { out } \Omega \\
\sigma_{i j}^{r}(x)=C_{i j k l}\left[\left(u_{k, l}+u_{l, k}\right) / 2-\varepsilon_{k l}^{*}\right] \quad \text { in } \Omega
\end{gathered}
$$

The entire calculation domain can be meshed into $M \times N \times L$ cuboid elements, each of which has a uniform eigenstrain and stress. After discretization, the stresses are given as the summation of cuboidal contributions in terms of the influence coefficients:

$$
\begin{aligned}
& \sigma_{i j}^{r}(\alpha, \beta, \gamma)= \\
& \frac{-\mu}{4 \pi(1-v)}\left(\sum_{\vartheta=0}^{L-1} \sum_{\eta=0}^{N-1 M-1} \sum_{\xi=0}^{(0)} T_{i j k l}^{(0)}(\alpha-\xi, \beta-\eta, \gamma-\vartheta) \cdot \varepsilon_{k l}^{p}(\xi, \eta, \vartheta)+\right. \\
& \sum_{\vartheta=0}^{L-1} \sum_{\eta=0}^{N-1 M-1} \sum_{\xi=0}^{(1)} T_{i j k l}^{(1)}(\alpha-\xi, \beta-\eta, \gamma+\vartheta) \cdot \varepsilon_{k l}^{p}(\xi, \eta, \vartheta)+ \\
& z \sum_{\vartheta=0}^{L-1} \sum_{\eta=0}^{N-1 M-1} \sum_{\xi=0}^{(2)} T_{i j k l}^{(2)}(\alpha-\xi, \beta-\eta, \gamma+\vartheta) \cdot \varepsilon_{k l}^{p}(\xi, \eta, \vartheta)+ \\
& \left.z^{2} \sum_{\vartheta=0}^{L-1} \sum_{\eta=0}^{N-1 M-1} \sum_{\xi=0}^{(3)} T_{i j k l}^{(3)}(\alpha-\xi, \beta-\eta, \gamma+\vartheta) \cdot \varepsilon_{k l}^{p}(\xi, \eta, \vartheta)\right)
\end{aligned}
$$

Here, $\alpha, \beta, \gamma$ denote the site of the element of interest, and $\xi, \eta, \vartheta$ are the element indices along the $x-, y$-, and $z$-directions. $T_{i j k l}^{(0)}, T_{i j k l}^{(1)}, T_{i j k l}^{(2)}, T_{i j k l}^{(3)}$ are the influence coefficients denoting the mapping relation from the unit eigenstrain to the eigenstress at a specific element. The explicit forms of the influence coefficients can be found in the appendices of Refs. [39, 51]. Thus, the discrete form of operation $Y$ for obtaining the eigenstress is determined by Eq. (13).

The calculation of the eigen-displacement can also be arranged into the form of an influence coefficient:

$$
\begin{aligned}
& u_{3}^{r}(x, y)= \\
& -\frac{1}{2 \pi} \sum_{\vartheta=0}^{L-1} \sum_{\eta=0}^{N-1 M-1} \sum_{\xi=0} D_{3 k l}(\alpha-\xi, \beta-\eta,-\vartheta) \cdot \varepsilon_{k l}^{*}(\xi, \eta, \vartheta)
\end{aligned}
$$

The explicit forms of the terms in Eq. (14) can be found in Ref. [52]. The calculation of the eigenstress and deformation involves three-dimensional discrete convolution, for which 3D-DC-FFT [53, 54] can be applied to accelerate the computation. It should be noted that the eigenstress calculation may be performed many times depending on the iterations of the CGM required to solve the equivalent eigenstrain.

\subsection{Damage evolution}

Butterfly formation is closely related to microstructural alterations, which can be described by the degradation of material properties [33]. Continuum damage mechanics (CDM) provides a potential method for evaluating the damage evolution and material deterioration. The phase transition from martensite to ferrite during butterfly formation is assumed to be represented by the damage variable $D$. In CDM theory, the stress-strain behavior is modified by the damage as follows [55]:

$$
\sigma_{i j}=C_{i j k l}\left(I_{m n k l}-D_{m n k l}\right) \varepsilon_{m n}
$$

The damage is reduced to a scalar variable under the assumption of isotropy. The damage-coupled constitutive equation then leads to the equivalent elastic modulus $C_{i j k l}^{*}=C_{i j k l}(1-D)$. Based on the results of nanoindentation tests, Moghaddam et al. [33] determined the elastic modulus of butterfly wings to be $C_{i j k l}^{*}=0.9 C_{i j k l}$. Herein, the same value of the equivalent elastic modulus is applied, and the critical damage value is set to $D^{*}=0.1$ accordingly. It is assumed that the element of interest is transformed and that no more damage occurs after the damage accumulation reaches the critical value. In the elastic 
contact state, the evolution of the damage variable $D$ can be described by [55]:

$$
\frac{\mathrm{d} D}{\mathrm{~d} N}=\left[\frac{\Delta \tau_{\mathrm{e}}}{\tau_{\mathrm{r}}(1-D)}\right]^{m}
$$

where $\tau_{\mathrm{r}}$ is the reference shear stress and $m$ denotes the stress component related to the Weibull slope. The parameters for the widely used AISI 52100 bearing steel taken from published works $[44,45]$ are $m=10.1$, $\tau_{\mathrm{r}}=6,113 \mathrm{MPa}$.

The butterfly is a typical shear stress-dominated RCF failure mode. Alternating shear stress is the primary driving factor for butterfly formation [2]. The effect of the mean stress cannot be neglected because the stress disturbance around the inclusion is asymmetrical. To incorporate the effects of both the mean stress and the stress amplitude during the rolling contact process, the equivalent stress is represented by [32]:

$$
\Delta \tau_{\mathrm{e}}=\tau_{\mathrm{alt}}+\left|\tau_{\mathrm{m}}\right|
$$

where $\tau_{\text {alt }}$ is the amplitude of the alternating shear stress, and $\tau_{\mathrm{m}}$ is the mean shear stress. The jump-incycle method is introduced to capture the cyclic loading state intrinsic to high-cycle fatigue. In this procedure, it is assumed that the stress range of each element changes very little over a specific contact cycle of $\Delta N$ [56-58]. Within the given finite cycles, the damage increment is given by

$$
\Delta D=\Delta N\left(\frac{\mathrm{d} D}{\mathrm{~d} N}\right)
$$

The contact solver is executed at every contact location. At the end of the rolling passage, the equivalent stress is extracted from the stress history, and the damage rate $\mathrm{d} D / \mathrm{d} N$ is calculated. Then, the total damage accumulation of each element is determined as

$$
D_{i+1}=D_{i}+\Delta D_{i=1}
$$

The local elastic modulus of the matrix is updated, and the heterogeneity element is embedded in the damage site. The next rolling passage is repeated using the updated elastic modulus. To reduce the computing cost, the damage threshold is set as $D_{t}=0.001$. Once the total damage accumulation of the element reaches the critical value, the transformed elements are added to the damage location of the butterfly map.

\section{Numerical simulation}

\subsection{Parameter determination}

The simulation was performed based on the results of the contact fatigue tests reported in a previous study [44]. The tests were conducted on a three-rings-on-roller test rig, as shown in Fig. 4(a). The diameters of the roller and rings were $12 \mathrm{~mm}$ and $54 \mathrm{~mm}$, respectively.
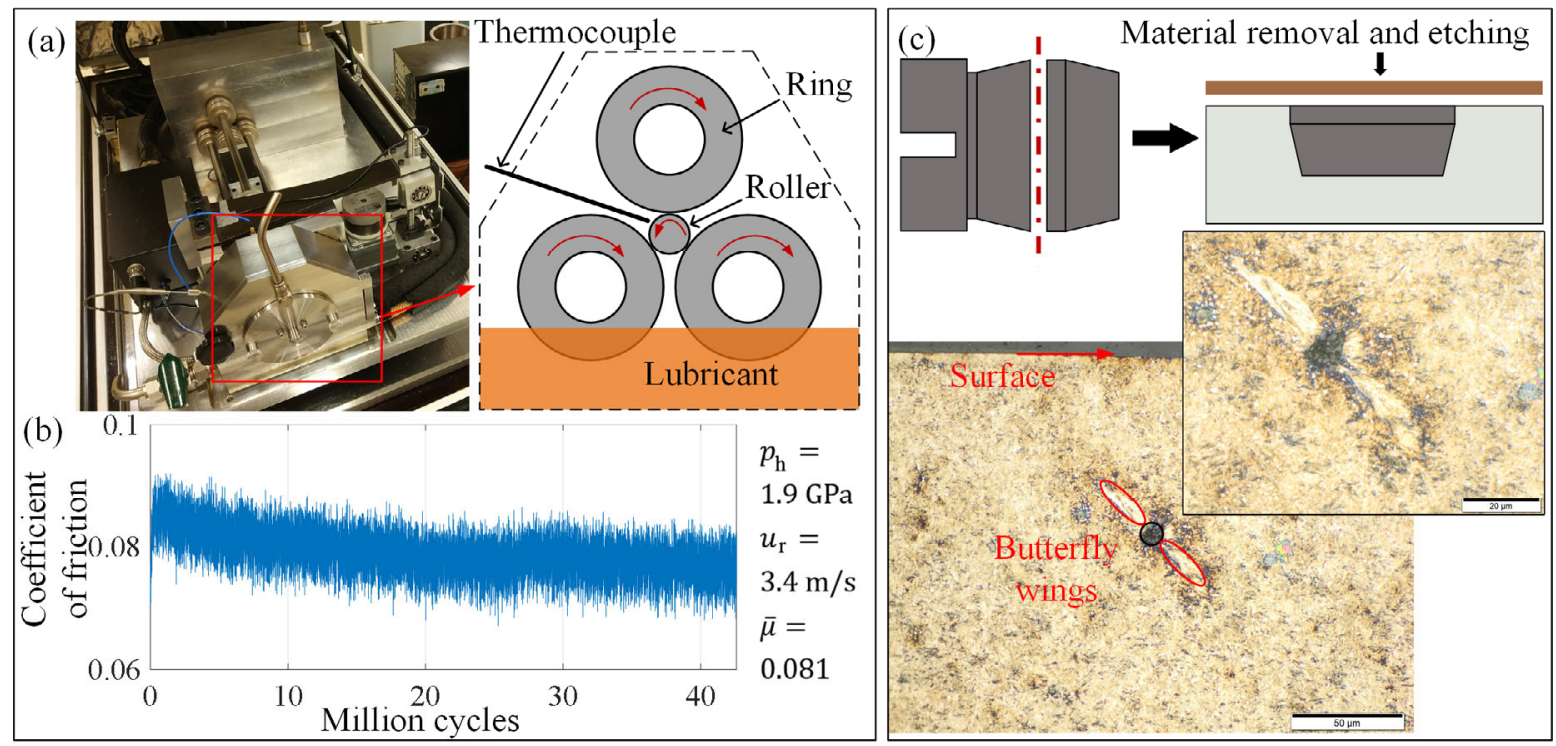

Fig. 4 (a) Three-rings-on-roller RCF tester; (b) measured coefficient of friction; (c) optical image of butterfly after section and etching. 
The material of the rings and roller was AISI 52100 through hardening tempered martensitic steel. The hardness of the roller and rings were 60 and $63 \mathrm{HRC}$, respectively. The Poisson's ratio and elastic modulus of the material matrix were $210 \mathrm{GPa}$ and 0.3 , respectively. The applied load in the test was approximately $500 \mathrm{~N}$, for which a maximum Hertzian pressure of $p_{\mathrm{h}}=1.9 \mathrm{GPa}$ and contact half width of $b=0.164 \mathrm{~mm}$ were maintained. The tests were performed under different slide-to-roll ratios and rolling speeds, and the roller was sectioned and inspected to identify the sub-surface-initiated cracks. Figures 4(b) and 4(c) show the results of one test in which the butterfly was identified. The coefficient of friction is shown in Fig. 4(b). The mean value throughout the test was $\bar{\mu}=0.081$, and the fatigue life was 42 million cycles. Herein, the coefficient of friction in the simulation was set to 0.08 .

To simulate the test, the contact between the roller and rings was simplified into a three-dimensional line contact in which a rigid roller contacted with a deformable plane containing an inhomogeneous inclusion. The load and geometry conditions were the same as those of the RCF test. The Poisson's ratio and elastic modulus of the inclusion were set as $389 \mathrm{GPa}$ and 0.25 , respectively, to be consistent with those of $\mathrm{Al}_{2} \mathrm{O}_{3}$, the most common type of oxide inclusion in 52100 bearing steel [59]. A spherical inclusion with the radius of $0.2 b$ was set at the depth of $z_{d}=0.5 b$ in the baseline case. The computation domain of $6 b \times 2 b \times b$ was discretized into a $193 \times 65 \times 33$ grid . The movement of the roller from $x=-2 b$ to $x=2 b$ was decomposed into 17 contact steps. The cyclic loading was applied in the contact procedure via the jump-in-cycle method. The material properties and damage accumulation were updated for every loading block. The number of loading blocks was determined based on $\Delta N$ and the tested fatigue life.

\subsection{Numerical verification}

To verify the computing accuracy and efficiency of the developed model, a comparison between the SAM solution and FEM results was conducted using the commercial software ABAQUS version 6.14. To remove the impact of the free boundary, a two-dimensional (2D) FE model and a three-dimensional (3D) quarter
FE model were compared with the SAM solutions to evaluate the computing accuracy and efficiency, respectively. The geometry conditions of the $2 \mathrm{D}$ model matched those of the actual contact. A $12 \mathrm{~mm}$-diameter roller containing a rigid inclusion was loaded on the $54 \mathrm{~mm}$-diameter ring, as shown in Fig. 5(a). The fine mesh area in the region around the inclusion was partitioned with a local seed size of $0.0025 \mathrm{~mm}$, and the element type was the eight-node plane strain reduced integration element (CPE8R). The 3D model was simplified as the contact pair of an analytical rigid roller with an equivalent radius of $4.9 \mathrm{~mm}$ and a quarter deformable cuboid based on Hertzian contact theory, as shown in Fig. 5(b). The size of the deformable body was fixed as $10 b \times 6 b \times 10 b$ along the $x-, y$-, and $z$-directions. The local seed size around the inclusion was set to $0.005 \mathrm{~mm}$, which is close to the SAM element size $(b / 32)$. The total number of elements in the 3D model was 401,680 , which is approximately half the number of SAM elements $(413,985)$. The simulation was performed on a platform with an Intel(R) Core ${ }^{\mathrm{TM}} \mathrm{i} 5-8500$ processor @ $3.00 \mathrm{GHz}$ (6 cores, 6 threads) and 16 GB memory.

The von Mises stress for a square and a circular inclusion are compared in Fig. 5(d) and Fig. 5(e), respectively. The stress distributions of the SAM solutions agree well with the FEM results. The maximum stress in Fig. 5(d) obtained by SAM and FEM are 1,637.5 MPa and 1,642 MPa, respectively. In Fig. 5(e), several stress peaks can be found on the edge of the inclusion. These peaks resulted from the non-smooth interface of the circle for the discrete grids. The peaks would not affect the accuracy of the current model because the stress and damage within the inclusion are not considered in the work. The computation time for the 3D model is shown in Fig. 5(c). The two meshing schemes of $385 \times 129 \times 65$ and $97 \times 33 \times 17$ were implemented to highlight the computation efficiency of current model. The results show that for the specific 3D contact problem considered in this work, the computing time for the developed SAM model was only $4.61 \%$ that of the FEM model. Considering the characteristics of rolling contact and the cyclic loading process, dozens of contact timesteps are required to simulate the damage evolution. The developed SAM model therefore has significant computing efficiency advantages. 


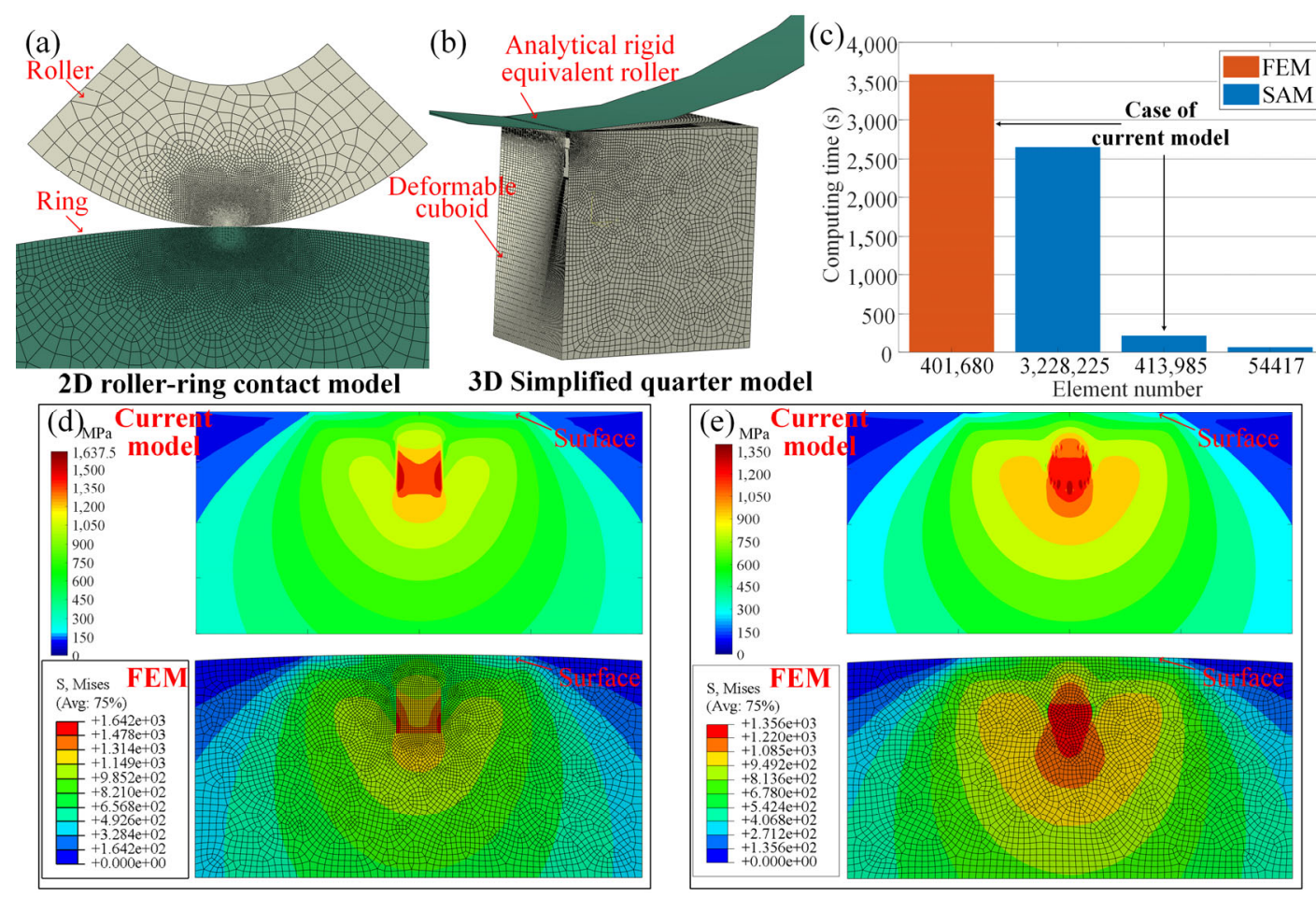

Fig. 5 Numerical verification of developed model: (a) 2D roller-ring contact FE model, (b) simplified 3D quarter FE model, (c) computing time, (d) von Mises stress field for square inclusion, (e) von Mises stress field for circular inclusion.

\section{Results and discussion}

\subsection{Stress distribution and damage evolution}

The stress history (i.e., time sequence of stress) is considered to be a major driver of butterfly formation and premature contact fatigue $[20,60]$. To capture the variation in stress history, a rolling contact procedure was simulated, and the variation of the stress components was documented. Figure 6 shows the variations in the alternating shear stress $\tau_{x z}$ during a rolling contact cycle. The sign of the shear stress depends on the direction of the $z$-axis. In this work, the $z$-axis points towards the depth direction. The minimum and maximum shear stresses are marked in black across the contour plots. It can be readily seen that the extremum of shear stress appears near the inclusion as the contact point moves close to the center. The maximum shear stress in Figs. 6(b) and 6(c) is 432.28 and $432.18 \mathrm{MPa}$, respectively, and increases to
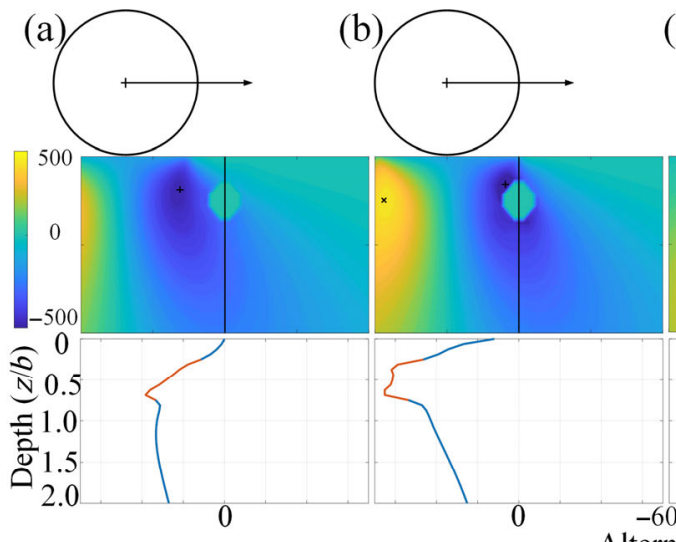

(c)

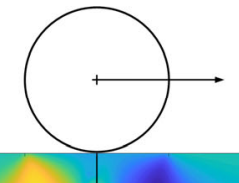

(d)

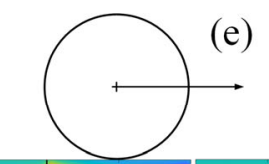

(e) Alternating shear stress $(\mathrm{MPa})$

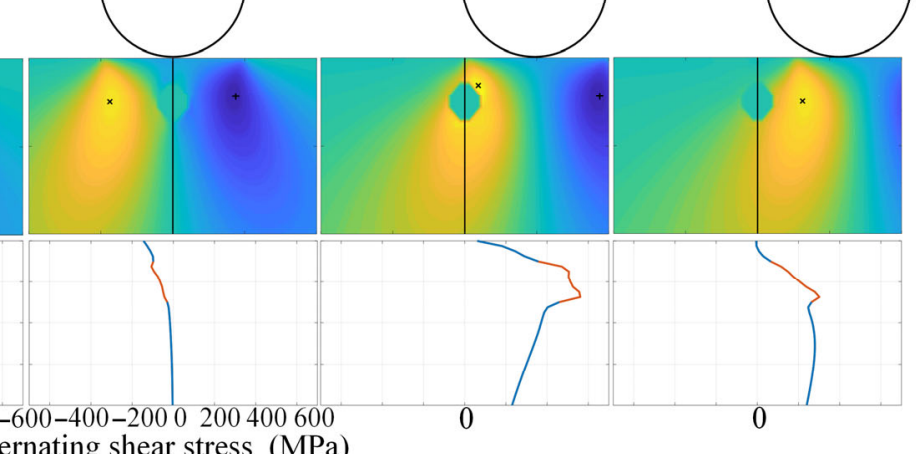

Fig. 6 Distribution of $\tau_{x z}$ in a rolling cycle. 
490.87 MPa in Fig 6(d). An obvious stress concentration near the inclusion can be observed when the highstress area passes over the inclusion.

The stress below the center point, which is denoted by the black solid line, is plotted in the second line of Fig. 6 . The red portion of the line represents the stress within the inclusion, which will not be presented in the remainder of this work because the damage accumulation in the inclusion was neglected. It can be readily seen that the site of the maximum alternating stress changes as the roller moves. When the contact point reaches the center (Fig. 6(c)), a small divergence of the shear stress below the center point can be observed. This value should be zero in the contact condition without the inclusion. The variation of the alternating shear stress during the rolling contact process is asymmetric owing to the inclusion. This indicates that a nonzero mean shear stress exists around the inclusion.

A further analysis of the shear stress history around the inclusions is presented in Fig. 7. It should be noted that the inclusion and surrounding areas present a non-smooth interface owing to the intrinsic discrete characteristics of the SAM. Figure 7(a) illustrates the composite shear stress in a rolling contact cycle. Two distinguishable regions can be found in the contours of the equivalent shear stress. These high-stress regions are diagonally distributed at the top left and bottom right of the inclusion. This distribution is consistent with the experimental observation that the growth of butterfly wings occurs at a specific angle to the rolling direction. Figure 7(b) shows the histories of the shear stress at the four corner positions during a complete rolling pass. The mean shear stresses at positions a-d are $17.13,96.68,16.98$, and $83.75 \mathrm{MPa}$, respectively. The alternating amplitudes at positions a-d are 482.09, 450.52 , 474.63 and $488.58 \mathrm{MPa}$, respectively. The variation of the equivalent stress components, that is, the alternating amplitude and mean shear stress, with the rotational angle $\kappa$ are shown in Fig. 7(c). It can be seen that the differences in the alternating amplitudes at different positions are limited, while the mean shear stress at the regions of the two wing regions is higher than that at the other regions. This implies that the mean shear stress is highly significant in butterfly initiation. It is hypothesized that the mean shear stress is related to surface traction, which introduces the disturbance of the shear stress around the inclusion.

The formation of butterfly wings during cyclic loading is shown in Fig. 8. The damaged zone, where microstructural alteration occurs, is represented by the small blue circles around the inclusion. The chronological order of butterfly formation illustrates the trend that the damage accumulates along a specific direction at an angle of approximately $45^{\circ}$. At the early stages, the left upper wing (which is closer to the surface) is more developed, as shown in Figs. 8(b) and 8(c). The two sides of the wings gradually propagate and become equally large as the damage accumulates. The emergence of the secondary wing is shown in Fig. 8(f). This process is consistent with the results reported by Moghaddam et al. [32].

The damaged elements are treated as inhomogeneous inclusions because of the degradation of the material properties (i.e., the reduction of elastic modulus). This deterioration process is accompanied by a variation in the stress field, as shown in Fig. 9. The deteriorated elements introduce stress disturbances in the surrounding area and cause further damage. The area of the lower wing is wider than that of the upper wing. One possible (a)

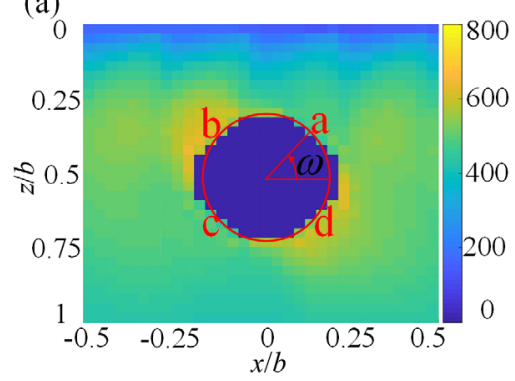

(b)

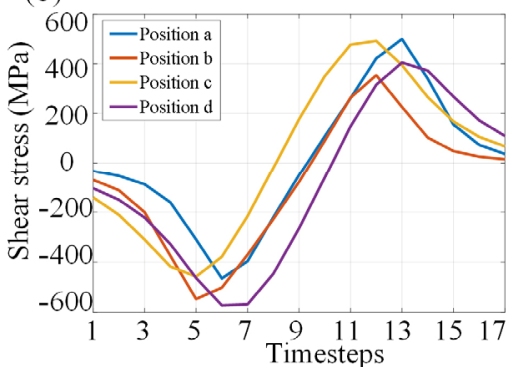

(c)

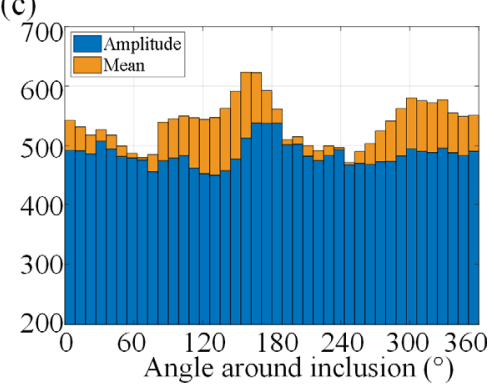

Fig. 7 Stress variation around inclusion at depth $z_{\mathrm{d}}=0.5 b$ : (a) equivalent shear stress, (b) stress profiles of $\tau_{x z}$, (c) amplitude and mean of shear stress versus rotational position. 

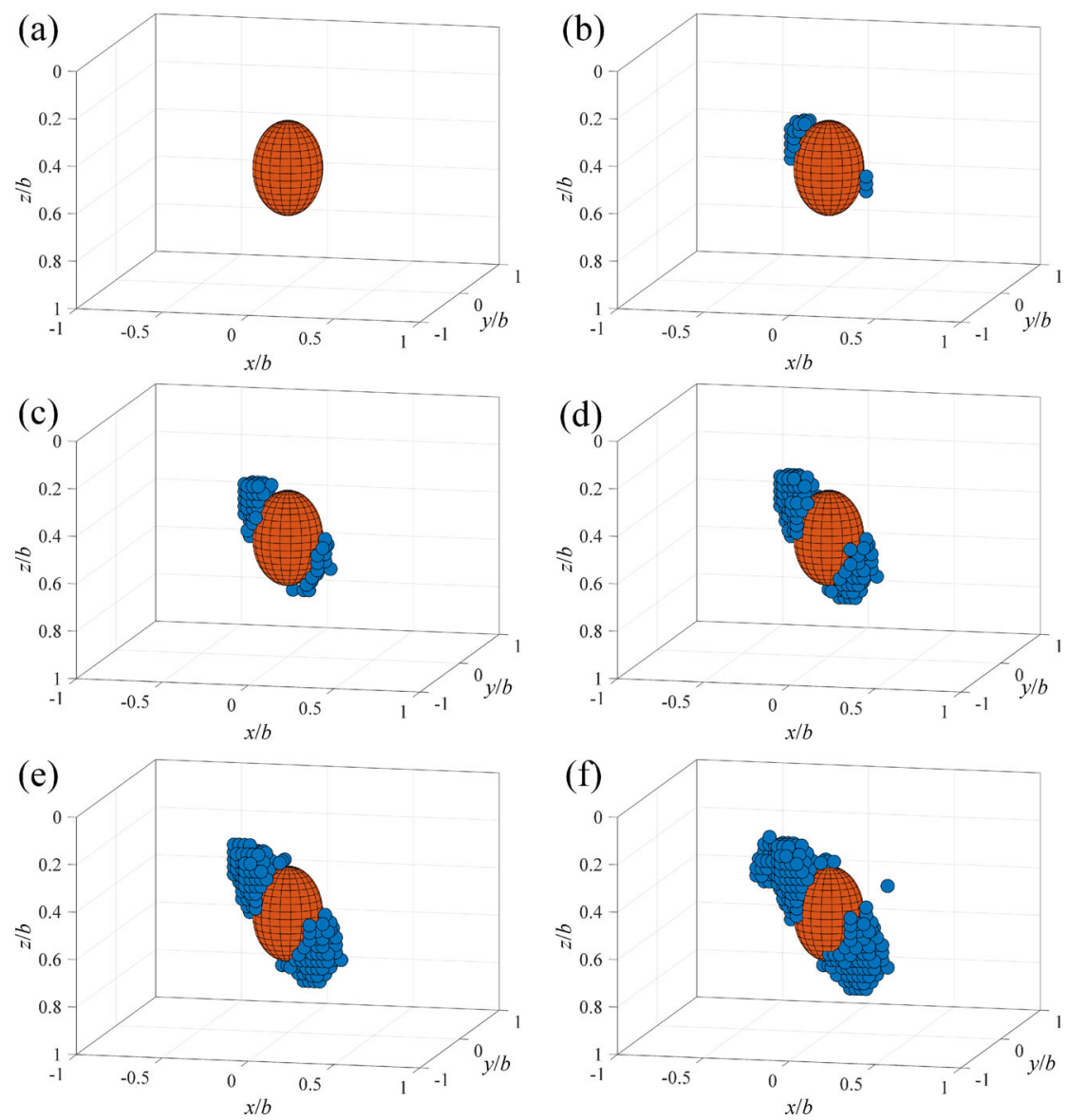

Fig. 8 Butterfly formation during cyclic loading at depth $z_{\mathrm{d}}=0.5 b$ : (a)-(f): $1^{\text {st }}-6^{\text {th }}$ loading block.
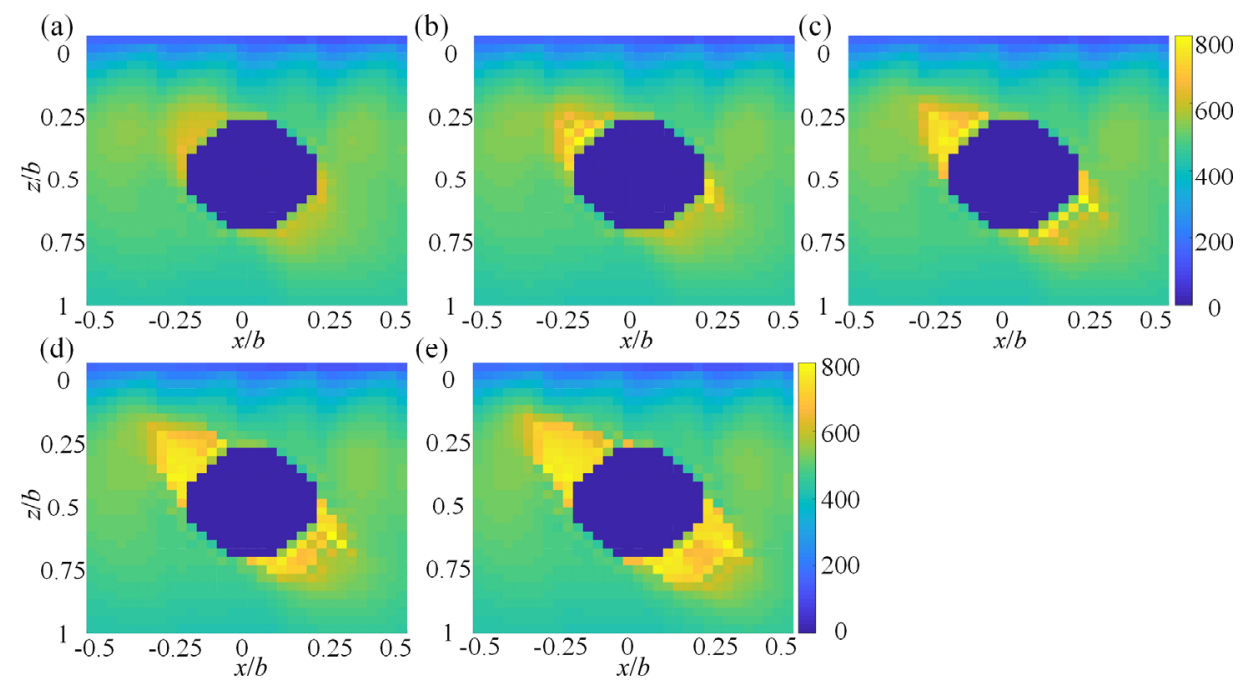

Fig. 9 Stress variation around inclusion during cyclic loading at depth $z_{\mathrm{d}}=0.5 b$ : (a)-(f): $1^{\text {st }}-6^{\text {th }}$ loading block.

reason for this is the larger area that experiences higher shear stress at the depth of the lower wing.

Figure 10 shows the distribution of the damage around the inclusion on different $X Z$ planes at $y=0$, $0.0625 b, 0.125 b, 0.1825 b$ and $0.25 b$. These figures present the same view as the serial sectioning of the tested samples. It can be readily seen that the damaged area deceases obviously with increasing distance from the mid-plane $(y=0)$. However, damage still occurs outside of the planes containing the inclusion. 

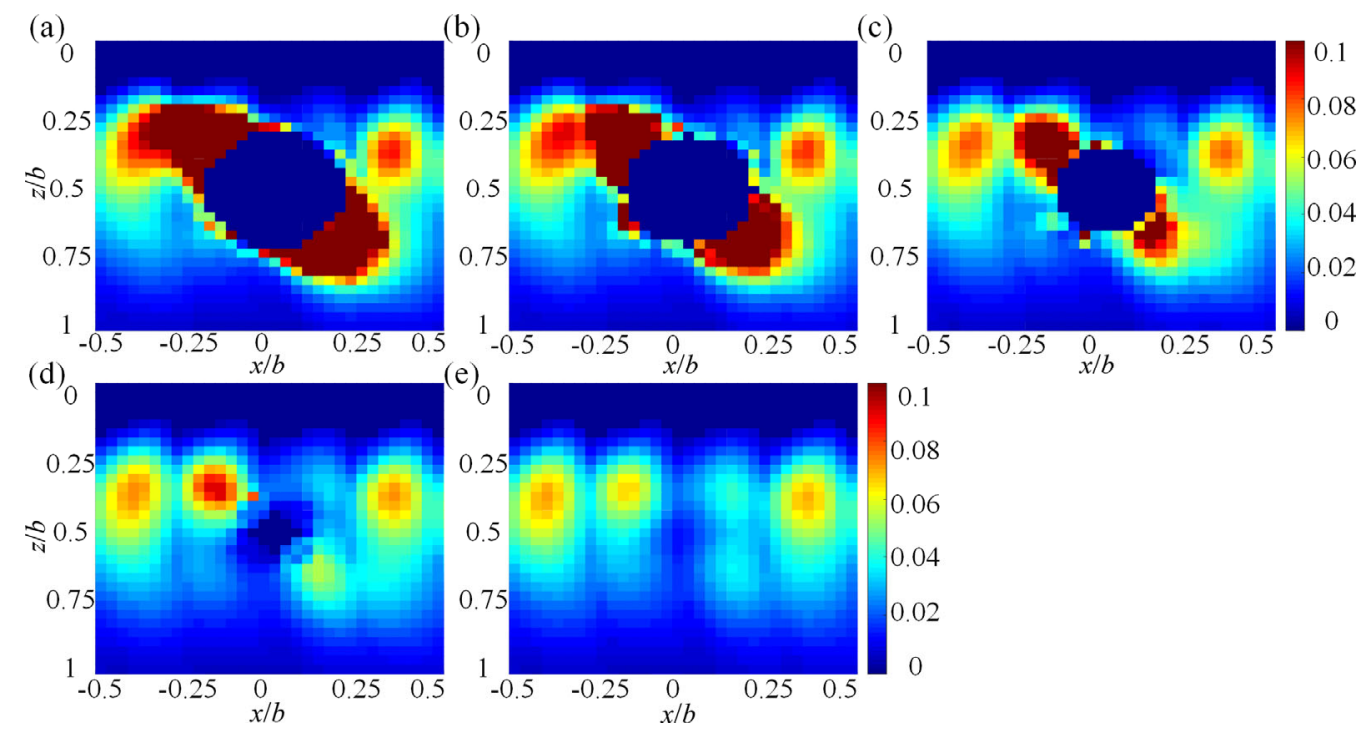

Fig. 10 Damage in different plane sections: (a) $y=0$, (b) $y=0.0625 b$, (c) $y=0.125 b$, (d) $y=0.1875 b$, (e) $y=0.25 b$.

This suggests that butterfly wings without inclusions might be observed in the sectioned samples. Especially for spherical inclusions, the wings away from the middle plane may grow beyond the extent of the inclusion if the loading cycle is high enough.

\subsection{Effect of inclusion depth}

The inclusion depth is a significant factor influencing butterfly formation. The stress concentration site is highly related to the inclusion depth. In this section, the depth is set as $z_{\mathrm{d}}=0.3 b-0.7 b$ to examine its effect on butterfly formation. Figure 11 shows the composite equivalent stresses at different depths at the first loading cycle. For the depth of $z_{\mathrm{d}}=0.3 b$ shown in Fig. 11(a), lower stress is found around the rotational angles $\kappa=30^{\circ}-60^{\circ}$ where the top right corner is close to the surface. The stress increases obviously in this area for $z_{\mathrm{d}}=0.5 b-0.7 b$. The main reason for this increase is the larger shear stress at this depth range. For a general Hertzian contact, the maximum alternating shear stress is located at the depth of $z_{\mathrm{d}}=0.5 b$. It can be seen that for the various depths, the mean shear stress is obviously higher around $\kappa=120^{\circ}$ and $\kappa=300^{\circ}$.

The 3D shapes of the butterflies formed at different depths are shown in Fig. 12. For $z_{\mathrm{d}}=0.3 b$ and $0.4 b$, it can be readily observed that the lower wing is

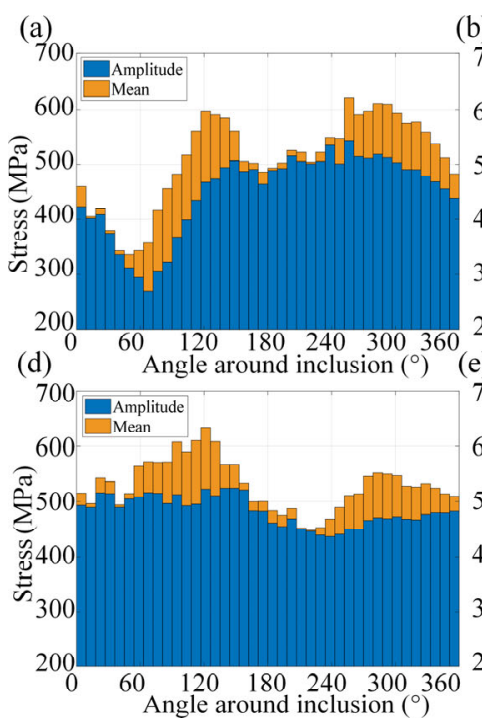

(b)
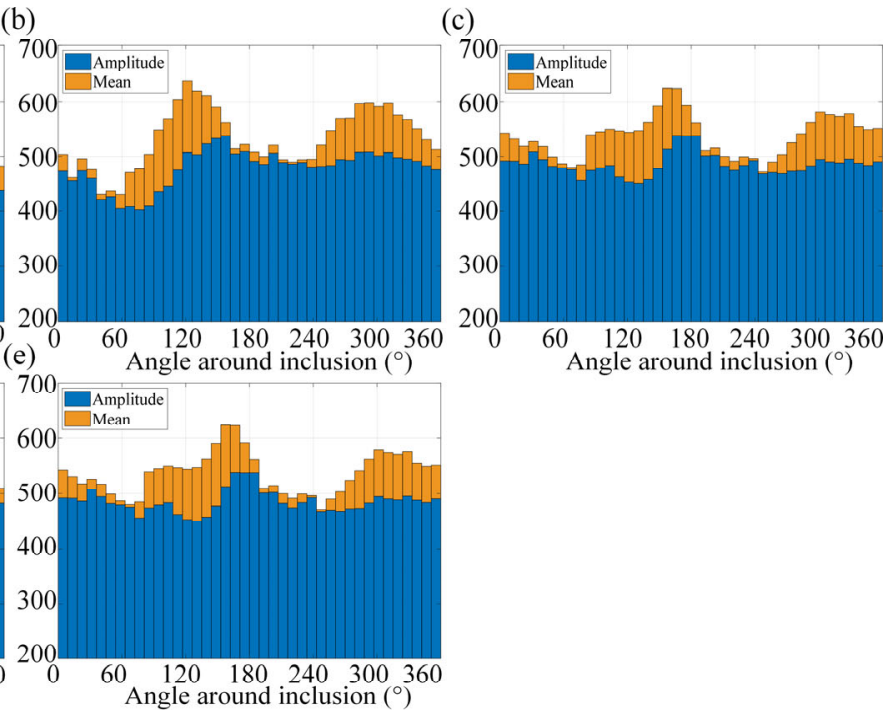

Fig. 11 Composite shear stress at different depths: (a) $z_{\mathrm{d}}=0.3 b$, (b) $z_{\mathrm{d}}=0.4 b$, (c) $z_{\mathrm{d}}=0.5 b$, (d) $z_{\mathrm{d}}=0.6 b$, (e) $z_{\mathrm{d}}=0.7 b$. 
larger by up to several times compared to the upper wing. The upper wing is more developed at $z_{\mathrm{d}}=0.6 \mathrm{~b}$ and $0.7 b$. Moreover, the initiated second pair of wings can also be found at the top right corner. It is also worth noting that when the inclusion is located at $z_{\mathrm{d}}=0.7 \mathrm{~b}$, the development of the lower wing is relatively limited. One possible reason for this is the decreased influence of the surface traction on the shear stress at deeper locations. The inclusion depth directly affects the initiation of the butterfly by changing the site of high stress concentration.

The stress field after butterfly formation is shown in Fig. 13. The sparsely distributed areas of higher stress, represented by the yellow elements, can be found at the upper wing for $z_{\mathrm{d}}=0.3 b-0.4 b$, and at the lower wing for $z_{\mathrm{d}}=0.6 b-0.7 b$. This suggests that the stress concentration is mild in the area where the wingspan is limited. The high-stress area on the other wing is more extended owing to the more developed butterfly wing. This observation partially explains the coupling between the damage accumulation and the stress variation. The stress concentration increases the damage, while the damaged area results in stress concentration, which leads to further damage accumulation in the surrounding area. This process is repeated under cyclic loading until crack initiation.
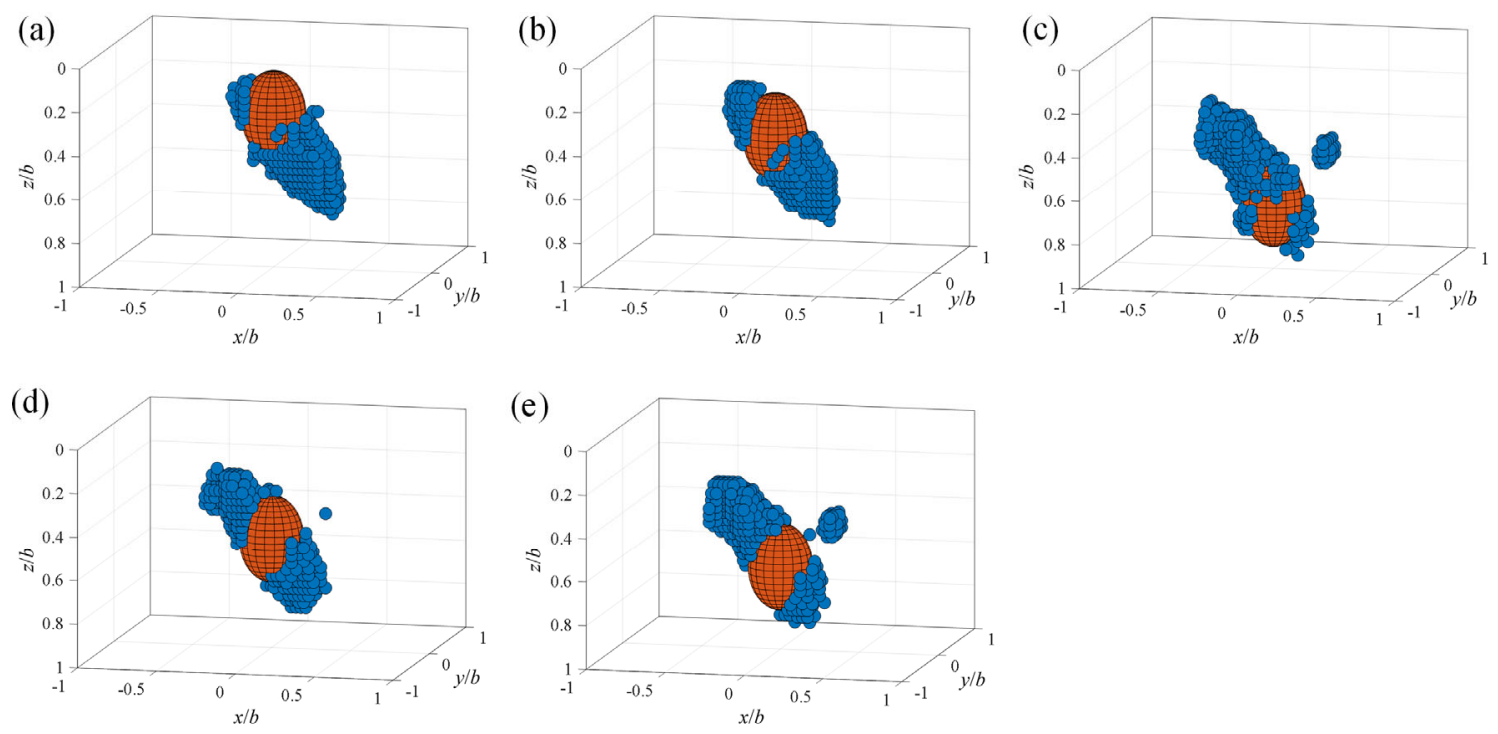

Fig. 12 Butterfly wings around inclusion at different depths: (a) $z_{\mathrm{d}}=0.3 b$, (b) $z_{\mathrm{d}}=0.4 b$, (c) $z_{\mathrm{d}}=0.5 b$, (d) $z_{\mathrm{d}}=0.6 b$, (e) $z_{\mathrm{d}}=0.7 b$.
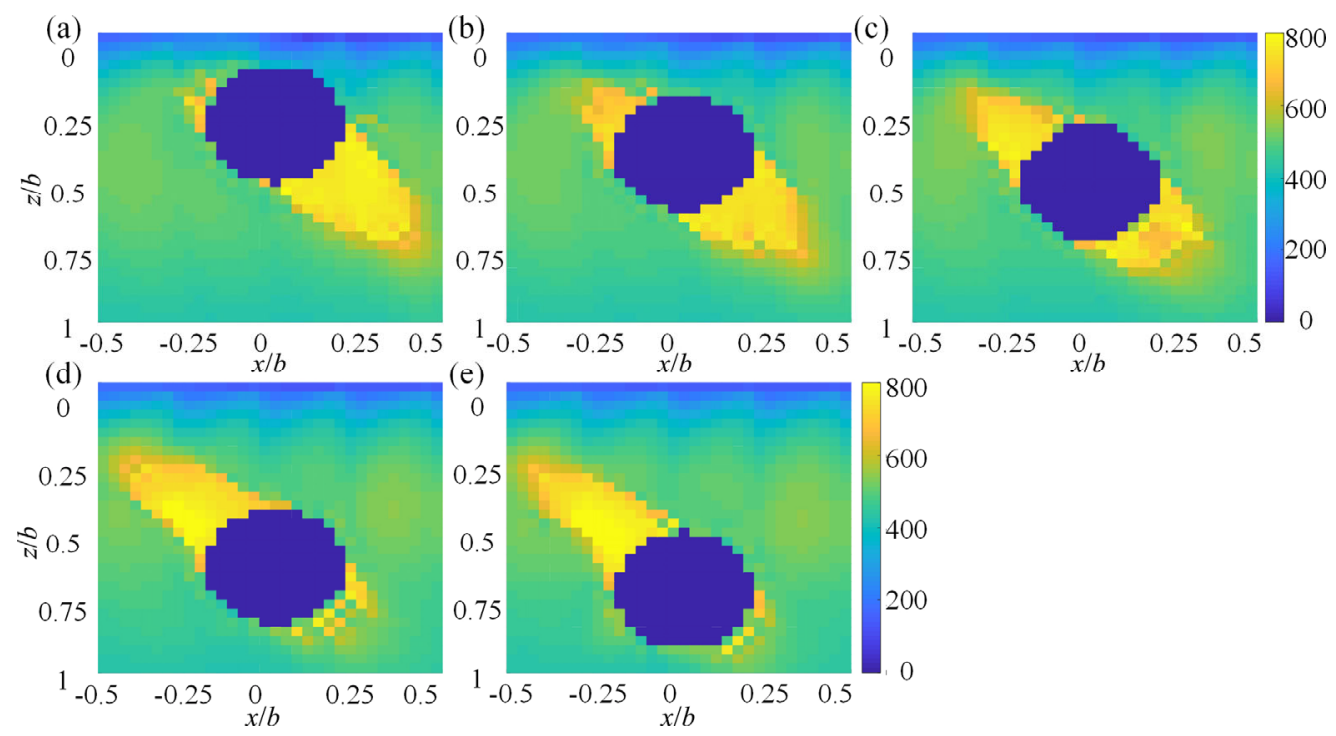

Fig. 13 Stress field around inclusion after butterfly formation: (a) $z_{\mathrm{d}}=0.3 b$, (b) $z_{\mathrm{d}}=0.4 b$, (c) $z_{\mathrm{d}}=0.5 b$, (d) $z_{\mathrm{d}}=0.6 b$, (e) $z_{\mathrm{d}}=0.7 b$. 


\subsection{Effect of inclusion orientation angle}

Irregular globular inclusions are a common type of material defects in engineering practice. In this section, an ellipsoid inclusion with the axial ratio of 2:1:1 at the depth of $z=0.5 b$ is assumed to investigate the influence of the inclusion orientation angle on butterfly formation. As shown in Fig. 14, the inclusion orientation angle $\omega$ is defined as the angle between the major axis of the ellipsoid and the rolling direction. Figure 15 illustrates butterfly formation under different $\omega$. It can be observed that the growth of the butterfly traces the same diagonal line for different direction angles. The upper wing is more developed at $\omega=0-60^{\circ}$, at

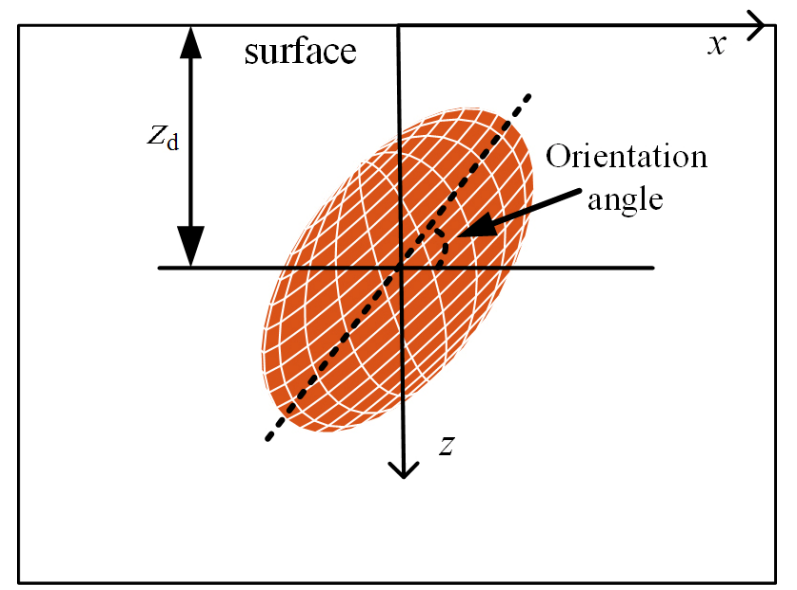

Fig. 14 Schematic diagram of inclusion orientation angle. which the long axis is closer to being parallel to the rolling direction. The initiation of the secondary pair of wings can be observed in these cases. At $\omega=120^{\circ}$ and $150^{\circ}$, butterfly wings grow regularly around both sides of the long axis.

Figure 16 further illustrates the number of damaged elements representing the propagation of butterfly wings. It can be readily seen that the damaged area is larger at acute direction angles compared to obtuse direction angles. One possible reason is the correspondence between the depth of the damaged regions and the depths at which the shear stress is higher. Another hypothesis is that the overall rolling direction might affect the stress history at different direction angles, which in turn changes the damage evolution process. This might explain the experimental observation of Bruce et al. [5] that longer cracks tend to be initiated from inclusions parallel to the raceway surface.

Figure 17 shows the stress fields for different orientation angles after butterfly formation. The areas of stress concentration area correspond well with the butterfly wings. At $\omega=30^{\circ}$ and $60^{\circ}$, discontinuous high stress distributions can be found in the area of lower wing. This is related to the scattered damaged elements observed in Figs. 15(b) and 15(c). At $\omega=120^{\circ}$ and $150^{\circ}$, the stress distribution and damaged area are more extensive and form a new ellipsoid-like
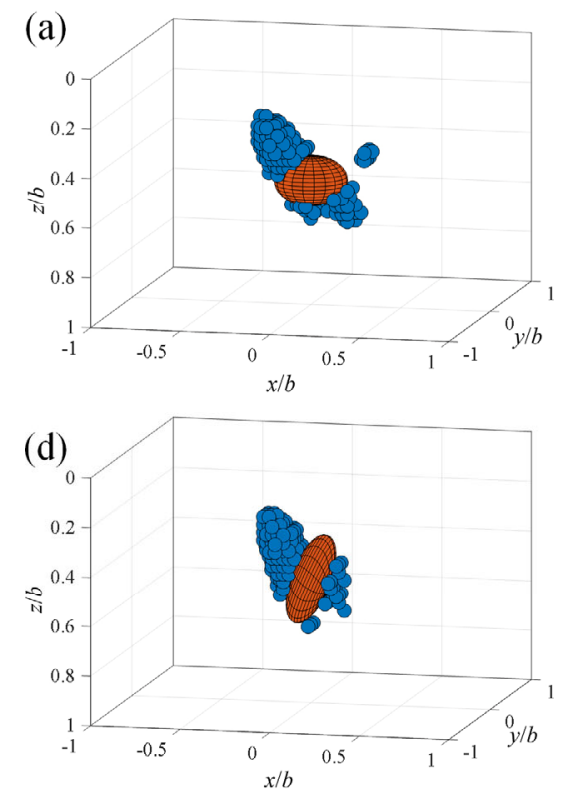
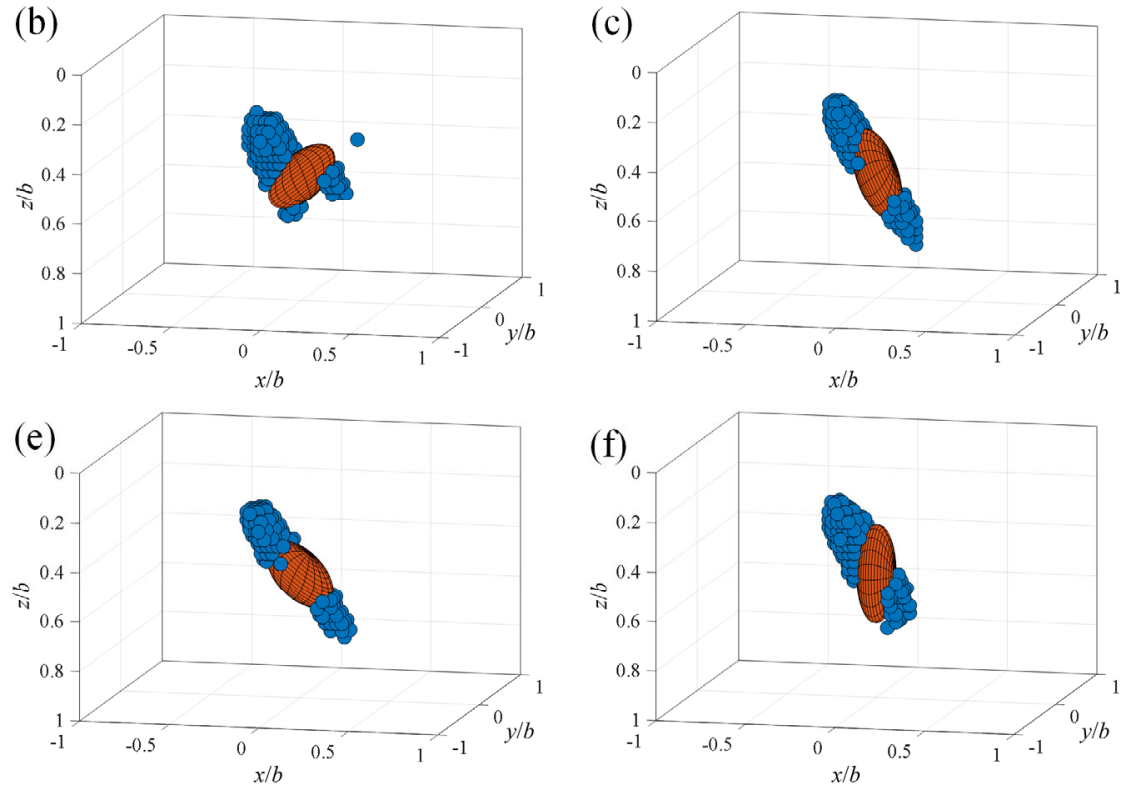

Fig. 15 Butterfly at different orientation angles: (a)-(f): $0,30^{\circ}, 60^{\circ}, 90^{\circ}, 120^{\circ}, 150^{\circ}$. 


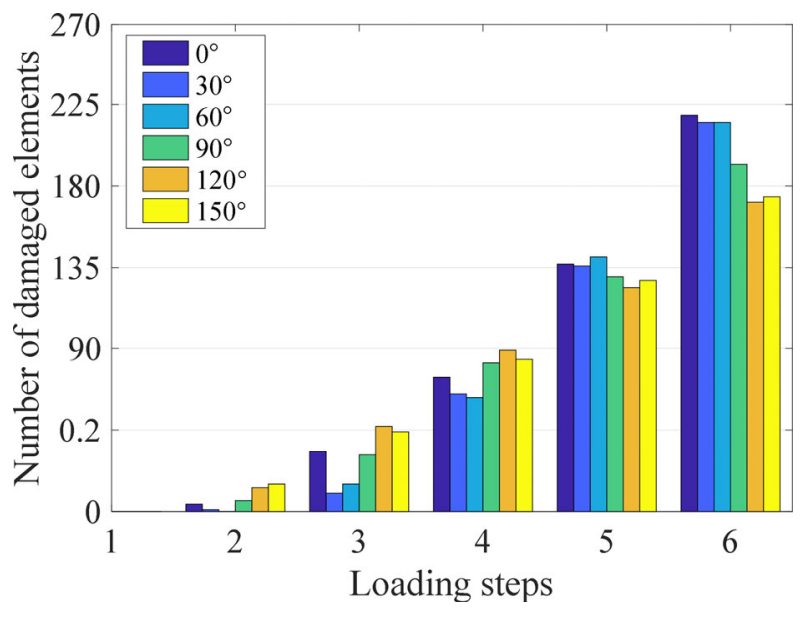

Fig. 16 Variation of number of damaged elements with orientation angle.

shape. The newly formed ellipsoid zone has a similar stress distribution to the original inclusion. It can be presumed that this similar shape would promote the further propagation of the damaged area, as per the butterfly wings (shown in Fig. 18) documented by Ganti et al. [61].

\section{Conclusions}

A semi-analytical 3D contact model incorporating inclusion and material property degradation was proposed to simulate butterfly formation. In the model, the elastic field caused by inhomogeneous and material deterioration is solved for by using a series of numerical methods. The accumulation of fatigue damage surrounding the inclusions is described by continuum damage mechanics. The coupling between the development of the damaged zone and the stress field is considered. The effects of the inclusion properties on the contact status and butterfly formation were discussed. The major conclusions are summarized as follows:

(1) The distribution of alternating shear stress is asymmetric around the inclusion. The stress analysis shows that the mean shear stress plays a significant role in butterfly formation.

(2) The depth of the inclusion directly affects the initiation of the butterfly by changing the site of high stress concentration. The coupling between the damage evolution and the stress variation around the inclusion is a major manifestation of butterfly formation.

(3) For ellipsoidal inclusions, the orientation angle $\omega$ significantly affects the formation of butterflies. The damaged area is larger at $\omega=0-60^{\circ}$ than at $\omega=90^{\circ}-150^{\circ}$.

This work provides a potential method for quantifying material defects and fatigue behavior in terms of the deterioration of material properties. In addition, multiple inclusions can be considered in the proposed model. However, some influencing factors, such as the thermal effect and imperfect bonding at the inclusion-matrix

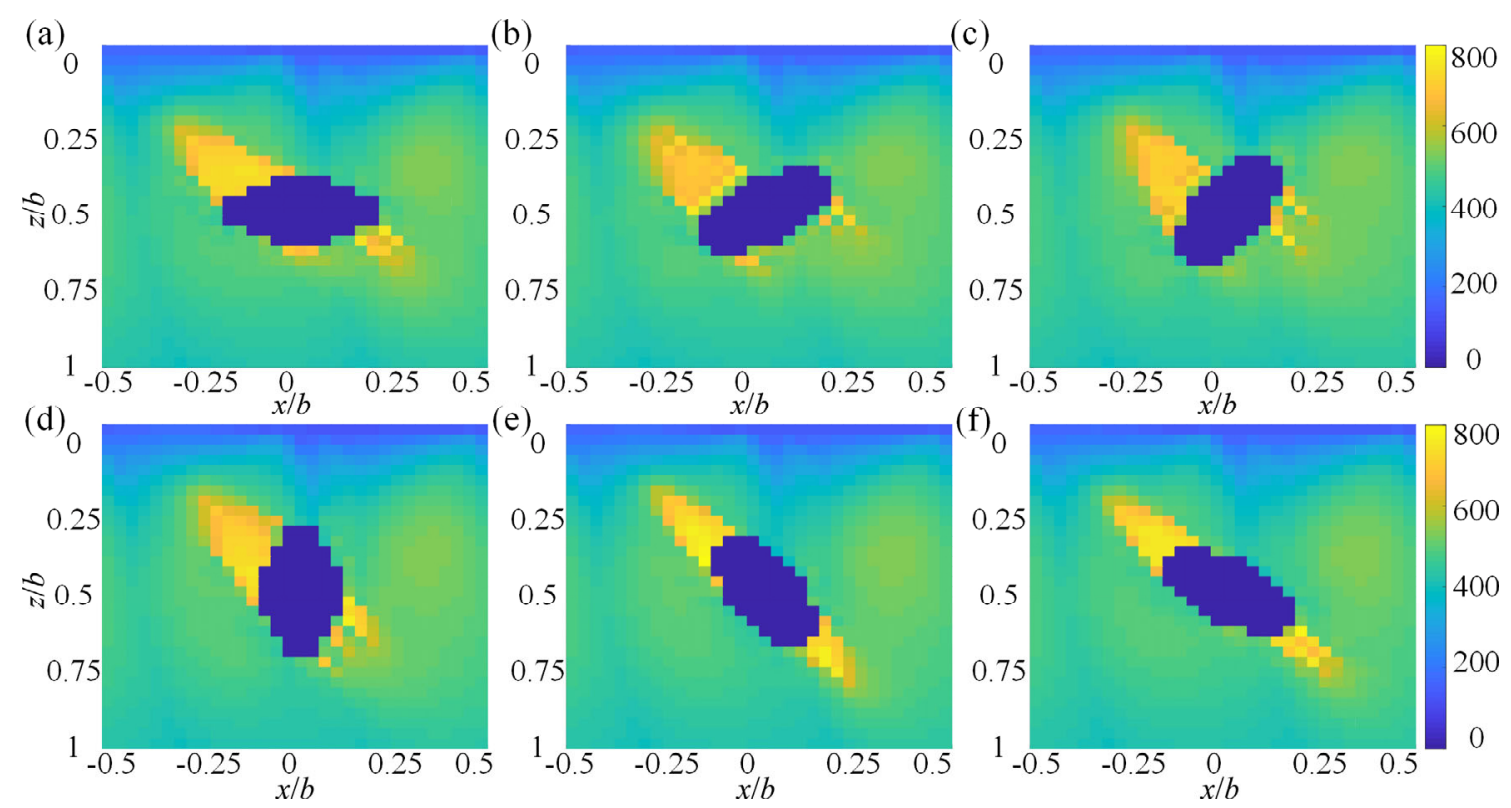

Fig. 17 Stress field around inclusion after butterfly formation in different orientation angles: (a)-(f): $\omega=0,30^{\circ}, 60^{\circ}, 90^{\circ}, 120^{\circ}, 150^{\circ}$. 


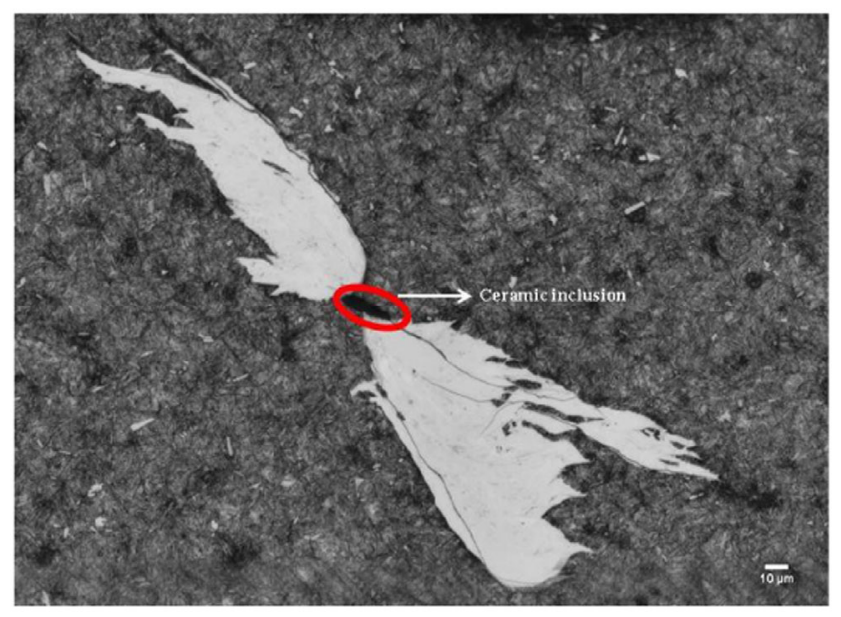

Fig. 18 Butterfly wings documented by Ganti et al. Reproduced with permission from Ref. [61], C) Elsevier 2018.

interface, still require further study.

\section{Authorship contribution statement}

Ye Zhou: Conceptualization, Methodology, Investigation, Writing-Original draft, Review \& Editing. Caichao Zhu: Project administration, Formal analysis, Supervision, Writing-Review \& Editing. Xiaojin Chen: Data acquisition and analysis, Writing-Review. Wei Ye: Validation, Formal analysis, Writing-Review.

\section{Acknowledgements}

This project is supported by National Natural Science Foundation of China (Nos. 52005057 and 51975063), and the Fundamental Research Funds for the Central Universities (Nos. 2020CDJQY-A069 and 2021CDJGXB 008).

\section{Appendix A}

The elastic matrix $C$ and its inverse $C^{-1}$ are as follows:

$$
\boldsymbol{C}=\left[\begin{array}{cccccc}
\lambda+2 \mu & \lambda & \lambda & & & \\
\lambda & \lambda+2 \mu & \lambda & & 0 & \\
\lambda & \lambda & \lambda+2 \mu & & & \\
& & & \mu & 0 & 0 \\
& 0 & & 0 & \mu & 0 \\
& & & 0 & 0 & \mu
\end{array}\right]
$$

$$
C^{-1}=\frac{1}{E}\left[\begin{array}{cccccc}
1 & -v & -v & & & \\
-v & 1 & -v & & 0 & \\
-v & -v & 1 & & & \\
& & & 2(1+v) & 0 & 0 \\
& 0 & & 0 & 2(1+v) & 0 \\
& & & 0 & 0 & 2(1+v)
\end{array}\right]
$$

Here, $E$ and $v$ are the elastic modulus and Poisson's ratio, respectively. The Lamé constants denoted as $\lambda$ and $\mu$ are $\lambda=\frac{E v}{(1+v)(1-2 v)}$ and $\mu=\frac{E}{2(1+v)}$.

\section{Appendix B}

The governing equation of the equivalent eigenstrain is

$$
\left(I-C C^{\phi-1}\right) \boldsymbol{Y}\left(\varepsilon^{*}\right)+C \varepsilon^{*}=\left(C C^{\phi-1}-I\right)\left[Y\left(\varepsilon^{p}\right)+\sigma^{0}\right]
$$

The left side of Eq. (B1) contains the only unknown $\varepsilon^{*}$, and the right can be determined using the given initial eigenstrain and load conditions. Eq. (B1) can then be simplified to a linear equation as follows:

$$
F \varepsilon^{*}=a
$$

The widely applied conjugate gradient method (CGM) [46] is used to solve Eq. (B2), as follows:

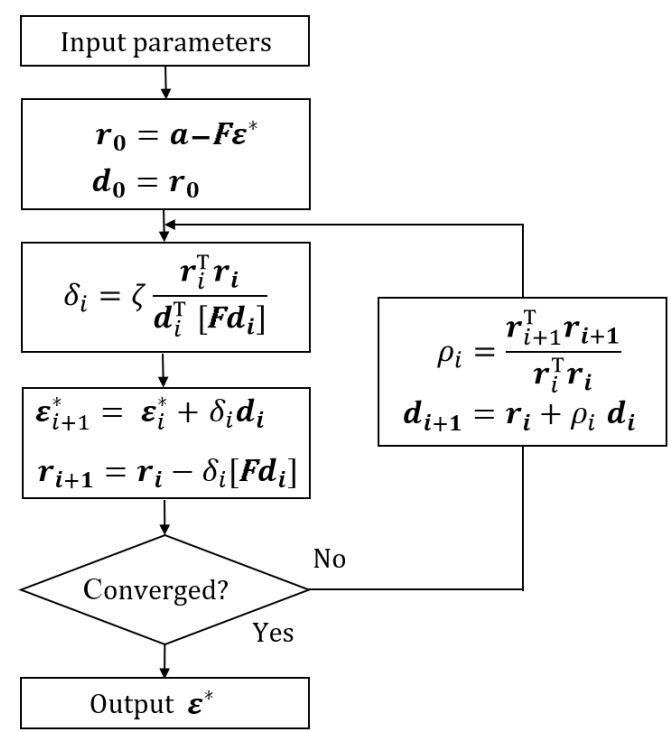

The vector $r$ denotes the conjugate residual, which is first calculated using a given initial value of $\varepsilon^{*}$. The 
conjugate direction $\boldsymbol{d}$ is determined in terms of the conjugate residual. The recommended values of the modification factor $\zeta$ for reducing the step size are $\zeta=0.5-0.8$. It should be noted that the vectors $\varepsilon^{*}, \boldsymbol{d}$, and $r$ all contain six components that can be solved for simultaneously in the iteration. In addition, the calculation of $\boldsymbol{F d}$ is the same as that of the left side of Eq. (B1), except that $\varepsilon^{*}$ is replaced with $d$.

Open Access This article is licensed under a Creative Commons Attribution 4.0 International License, which permits use, sharing, adaptation, distribution and reproduction in any medium or format, as long as you give appropriate credit to the original author(s) and the source, provide a link to the Creative Commons licence, and indicate if changes were made.

The images or other third party material in this article are included in the article's Creative Commons licence, unless indicated otherwise in a credit line to the material. If material is not included in the article's Creative Commons licence and your intended use is not permitted by statutory regulation or exceeds the permitted use, you will need to obtain permission directly from the copyright holder.

To view a copy of this licence, visit http://creativecommons.org/licenses/by/4.0/.

\section{References}

[1] Luyckx J. Hammering wear impact fatigue hypothesis WEC/irWEA failure mode on roller bearings. In Wind Turbine Tribology Seminar, Broomfield, CO, USA, 2011.

[2] Evans M H. An updated review: White etching cracks (WECs) and axial cracks in wind turbine gearbox bearings. Mater Sci Technol 32(11): 1133-1169 (2016)

[3] Grabulov A, Ziese U, Zandbergen H W. TEM/SEM investigation of microstructural changes within the white etching area under rolling contact fatigue and 3-D crack reconstruction by focused ion beam. Scripta Mater 57(7): 635-638 (2007)

[4] Gould B, Demas N G, Greco A C. The influence of steel microstructure and inclusion characteristics on the formation of premature bearing failures with microstructural alterations. Mater Sci Eng: A 751: 237-245 (2019)

[5] Bruce T, Rounding E, Long H, Dwyer-Joyce R S. Characterisation of white etching crack damage in wind turbine gearbox bearings. Wear 338-339: 164-177 (2015)
[6] Singh H, Pulikollu R V, Hawkins W, Smith G. Investigation of microstructural alterations in low- and high-speed intermediate-stage wind turbine gearbox bearings. Tribol Lett 65(3): 81 (2017)

[7] Neishi Y, Makino T, Matsui N, Matsumoto H, Higashida M, Ambai H. Influence of the inclusion shape on the rolling contact fatigue life of carburized steels. Metall Mater Trans A 44(5): 2131-2140 (2013)

[8] Jones A B. Metallographic observations of ball bearing fatigue phenomena. In Symposium on Testing of Bearings, West Conshohocken, PA, 1947.

[9] Styri H. Fatigue strength of ball bearing races and heattreated 52100 steel specimens. In Proceedings of American Society for Testing and Materials, 1951: 682-700.

[10] Evans M H, Wang L, Jones H, Wood R J K. White etching crack (WEC) investigation by serial sectioning, focused ion beam and 3-D crack modelling. Tribol Int 65: 146-160 (2013)

[11] Evans M H, Richardson A D, Wang L, Wood R J K, Anderson W B. Confirming subsurface initiation at non metallic inclusions as one mechanism for white etching crack (WEC) formation. Tribol Int 75: 87-97 (2014)

[12] Gould B, Greco A, Stadler K, Vegter E, Xiao X H. Using advanced tomography techniques to investigate the development of White Etching Cracks in a prematurely failed field bearing. Tribol Int 116: 362-370 (2017)

[13] Al-Tameemi H A, Long H, Dwyer-Joyce R S. Initiation of sub-surface micro-cracks and white etching areas from debonding at non-metallic inclusions in wind turbine gearbox bearing. Wear 406-407: 22-32 (2018)

[14] Lai J B, Stadler K. Investigation on the mechanisms of white etching crack (WEC) formation in rolling contact fatigue and identification of a root cause for bearing premature failure. Wear 364-365: 244-256 (2016)

[15] Gould B, Demas N G, Pollard G, Rydel J J, Ingram M, Greco A C. The effect of lubricant composition on white etching crack failures. Tribol Lett 67: 7 (2019)

[16] Gould B, Paladugu M, Demas N G, Greco A C, Hyde R S. Figure the impact of steel microstructure and heat treatment on the formation of white etching cracks. Tribol Int 134: 232-239 (2019)

[17] Roy S, Gould B, Zhou Y, Demas N G, Greco A C, Sundararajan S. Effect of retained austenite on white etching crack behavior of carburized AISI 8620 steel under boundary lubrication. Tribol Lett 67(2): 40 (2019)

[18] Fu H W, Rydel J J, Gola A M, Yu F, Geng K, Lau C, Luo H W, Rivera-Díaz-del-castillo P E J. The relationship between $100 \mathrm{Cr} 6$ steelmaking, inclusion microstructure and rolling contact fatigue performance. Int J Fatigue 129: 104899 (2019) 
[19] Lund T B. Sub-surface initiated rolling contact fatigueinfluence of non-metallic inclusions, processing history, and operating conditions. In Bearing Steel Technology, 8th Volume: Developments in Rolling Bearing Steels and Testing, West Conshohocken, PA, 2010: 81-96.

[20] Manieri F, Stadler K, Morales-Espejel G E, Kadiric A. The origins of white etching cracks and their significance to rolling bearing failures. Int J Fatigue 120: 107-133 (2019)

[21] Salehizadeh H, Saka N. The mechanics of crack initiation at hard particles in rolling line contacts. J Tribol 114(2): 341-347 (1992)

[22] Melander A. A finite element study of short cracks with different inclusion types under rolling contact fatigue load. Int J Fatigue 19(1): 13-24 (1997)

[23] Yamakawa K, Kizawa K, Oguma N, Kida K. Influence of subsurface defects on stress distribution under rolling contact conditions. Koyo Eng J English Ed (166E): 24-28 (2005)

[24] Raje N, Sadeghi F, Rateick Jr R G, Hoeprich M R. Evaluation of stresses around inclusions in hertzian contacts using the discrete element method. J Tribol 129(2): 283-291 (2007)

[25] Ueda K, Goshima T, Ishihara S, Shimizu M. Initial crack growth emanating from an inclusion due to rolling/sliding contact. J Therm Stresses 31(2): 107-126 (2007)

[26] McDowell D L. Simulation-based strategies for microstructuresensitive fatigue modeling. Mater Sci Eng: A 468-470: 4-14 (2007)

[27] Alley E S, Neu R W. Microstructure-sensitive modeling of rolling contact fatigue. Int J Fatigue 32(5): 841-850 (2010)

[28] Zhou H, Wei P T, Liu H J, Zhu C C, Lu C, Deng G Y. Roles of microstructure, inclusion, and surface roughness on rolling contact fatigue of a wind turbine gear. Fatigue Fract Eng Mater Struct 43(7): 1368-1383 (2020)

[29] Wang W, Liu H J, Zhu C C, Wei P T, Tang J Y. Effects of microstructure on rolling contact fatigue of a wind turbine gear based on crystal plasticity modeling. Int J Fatigue 120: 73-86 (2019)

[30] Wang W, Liu H J, Zhu C C, Wei P T, Wu W. Micromechanical analysis of gear fatigue-ratcheting damage considering the phase state and inclusion. Tribol Int 136: 182-195 (2019)

[31] Cerullo M, Tvergaard V. Micromechanical study of the effect of inclusions on fatigue failure in a roller bearing. Int J Struct Integr 6(1): 124-141 (2015)

[32] Mobasher Moghaddam S, Sadeghi F, Weinzapfel N, Liebel A. A damage mechanics approach to simulate butterfly wing formation around nonmetallic inclusions. J Tribol 137(1): 011404 (2015)

[33] Moghaddam M S, Sadeghi F, Paulson K, Weinzapfel N, Correns M, Bakolas V, Dinkel M. Effect of non-metallic inclusions on butterfly wing initiation, crack formation, and spall geometry in bearing steels. Int J Fatigue 80: 203-215 (2015)

[34] Guan J, Wang L Q, Zhang Z Q, Shi X J, Ma X X. Fatigue crack nucleation and propagation at clustered metallic carbides in M50 bearing steel. Tribol Int 119: 165-174 (2018)

[35] Jacq C, Nélias D, Lormand G, Girodin D. Development of a three-dimensional semi-analytical elastic-plastic contact code. J Tribol 124(4): 653-667 (2002)

[36] Nélias D, Antaluca E, Boucly V, Cretu S. A threedimensional semianalytical model for elastic-plastic sliding contacts. J Tribol 129(4): 761-771 (2007)

[37] Chaise T, Nélias D. Contact pressure and residual strain in 3D elasto-plastic rolling contact for a circular or elliptical point contact. J Tribol 133(4): 041402 (2011)

[38] Zhou K, Keer L M, Wang Q J. Semi-analytic solution for multiple interacting three-dimensional inhomogeneous inclusions of arbitrary shape in an infinite space. Int J Numer Methods Eng 87(7): 617-638 (2011)

[39] Liu S B, Jin X Q, Wang Z J, Keer L M, Wang Q. Analytical solution for elastic fields caused by eigenstrains in a half-space and numerical implementation based on FFT. Int J Plast 35: 135-154 (2012)

[40] Dong Q B, Zhou K. Modeling heterogeneous materials with multiple inclusions under mixed lubrication contact. Int $J$ Mech Sci 103: 89-96 (2015)

[41] Wang Z J, Jin X Q, Keer L M, Wang Q. Novel model for partial-slip contact involving a material with inhomogeneity. J Tribol 135(4): 041401 (2013)

[42] Zhou Q H, Jin X Q, Wang Z J, Wang J X, Keer L M, Wang Q. Numerical EIM with 3D FFT for the contact with a smooth or rough surface involving complicated and distributed inhomogeneities. Tribol Int 93: 91-103 (2016)

[43] Koumi K E, Chaise T, Nelias D. Rolling contact of a rigid sphere/sliding of a spherical indenter upon a viscoelastic half-space containing an ellipsoidal inhomogeneity. $J$ Mech Phys Solids 80: 1-25 (2015)

[44] Zhou Y, Zhu C C, Gould B, Demas N G, Liu H J, Greco A C. The effect of contact severity on micropitting: Simulation and experiments. Tribol Int 138: 463-472 (2019)

[45] Warhadpande A, Sadeghi F, Kotzalas M N, Doll G. Effects of plasticity on subsurface initiated spalling in rolling contact fatigue. Int J Fatigue 36(1): 80-95 (2012)

[46] Polonsky I A, Keer L M. A numerical method for solving rough contact problems based on the multi-level multisummation and conjugate gradient techniques. Wear 231(2): 206-219 (1999)

[47] Mura T. Micromechanics of Defects in Solids. 2nd edn. Dordrecht (Netherlands): Springer, 1987. 
[48] Chiu Y P. On the stress field due to initial strains in a cuboid surrounded by an infinite elastic space. J Appl Mech 44(4): 587-590 (1977)

[49] Zhou Y, Zhu C C, Liu H J, Song H L. Investigation of contact performance of case-hardened gears under plastoelastohydrodynamic lubrication. Tribol Lett 67(3): 92 (2019)

[50] Yu H Y, Sanday S C. Elastic field in joined semi-infinite solids with an inclusion. Proc Roy Soc A: Math Phys Eng Sci 434(1892): 521-530 (1991)

[51] Wang Z J, Jin X Q, Zhou Q H, Ai X L, Keer L M, Wang Q. An efficient numerical method with a parallel computational strategy for solving arbitrarily shaped inclusions in elastoplastic contact problems. J Tribol 135(3): 031401 (2013)

[52] Wang Z J, Jin X Q, Liu S B, Keer L M, Cao J, Wang Q. A new fast method for solving contact plasticity and its application in analyzing elasto-plastic partial slip. Mech Mater 60: 18-35 (2013)

[53] Liu S B, Wang Q. Studying contact stress fields caused by surface tractions with a discrete convolution and fast fourier transform algorithm. J Tribol 124(1): 36-45 (2002)

[54] Zhou K, Chen W W, Keer L M, Wang Q J. A fast method for solving three-dimensional arbitrarily shaped inclusions in a half space. Computer Methods Appl Mech Eng 198 (9-12): 885-892 (2009)

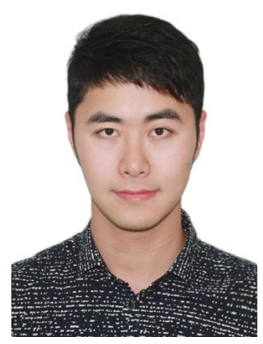

Ye ZHOU. He is currently working as an assistant research fellow in the State Key Laboratory of Mechanical Transmissions, Chongqing University,

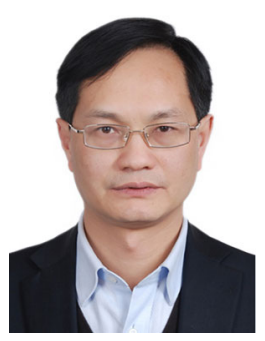

Caichao ZHU. He is a professor in the State Key Laboratory of Mechanical Transmissions, Chongqing University, China. He received his
[55] Lemaitre J, Desmorat R. Engineering Damage Mechanics: Ductile, Creep, Fatigue and Brittle Failures. Berlin, Heidelberg (Germany): Springer, 2005.

[56] Beyer T, Sadeghi F, Chaise T, Leroux J, Nelias D. A coupled damage model and a semi-analytical contact solver to simulate butterfly wing formation around nonmetallic inclusions. Int J Fatigue 127: 445-460 (2019)

[57] Walvekar A A, Sadeghi F. Rolling contact fatigue of case carburized steels. Int J Fatigue 95: 264-281 (2017)

[58] Morales-Espejel G E, Rycerz P, Kadiric A. Prediction of micropitting damage in gear teeth contacts considering the concurrent effects of surface fatigue and mild wear. Wear 398-399: 99-115 (2018)

[59] Hashimoto K, Fujimatsu T, Tsunekage N, Hiraoka K, Kida $\mathrm{K}$, Santos E C. Study of rolling contact fatigue of bearing steels in relation to various oxide inclusions. Mater Des 32(3): 1605-1611 (2011)

[60] Zhou Y, Zhu C C, Liu H J. A micropitting study considering rough sliding and mild wear. Coatings 9(10): 639 (2019)

[61] Ganti S, Turner B, Kirsch M, Anthony D, McCoy B, Trivedi H, Sundar V. Three-dimensional (3D) analysis of white etching bands (WEBs) in AISI M50 bearing steel using automated serial sectioning. Mater Charact 138: 11-18 (2018)

China. He received his Ph.D. degree from Chongqing University, China, in 2019. His research mainly relates to tribology and contact fatigue of mechanical elements.

Ph.D. degree from Chongqing University, China, in 1998. His research mainly relates to the intelligent design and dynamic analysis of transmission systems. 